\title{
Extended quantum criticality of low-dimensional superconductors near a spin-density-wave instability
}

\author{
A. Sedeki ${ }^{1}$, D. Bergeron ${ }^{1}$ and C. Bourbonnais ${ }^{1,2}$ \\ ${ }^{1}$ Regroupement Québecois sur les Matériaux de Pointe, Département de physique, \\ Université de Sherbrooke, Sherbrooke, Québec, Canada, J1K-2R1 and \\ ${ }^{2}$ Canadian Institute for Advanced Research, Toronto, Canada
}

(Dated: October 31, 2018)

\begin{abstract}
We use the renormalization group method to study normal state properties of quasi-onedimensional superconductors nearby a spin-density-wave instability. On the basis of one-loop scattering amplitudes for the quasi-one-dimensional electron gas, the integration of the renormalization group equations for the two-loop single particle Matsubara self-energy leads to a nonFermi-liquid temperature downturn of the momentum-resolved quasi-particle weight over most part of the Fermi surface. The amplitude of the downturn correlates with the entire instability line for superconductivity, defining an extended quantum critical region of the phase diagram as a function of nesting deviations of the Fermi surface. One also extracts the downward renormalization of interchain hopping amplitudes at arbitrary low temperature in the normal phase. By means of analytical continuation of the Matsubara self-energy, one-particle spectral functions are obtained with respect to both energy and temperature and their anomalous features analyzed in connection with the sequence of instability lines of the phase diagram. The quasi-particle scattering rate is found to develop an unusual temperature dependence, which is best described by the superimposition of a linear and quadratic $T$ dependences. The nonFermi-liquid linear- $T$ component correlates with the temperature scale $T_{c}$ of the superconducting instability over an extended range of nesting deviations, whereas its anisotropy along the Fermi surface is predicted to parallel the momentum profile of a d-wave pairing gap on the Fermi surface. We examine the implications of our results for low dimensional unconventional superconductors, in particular the Bechgaard salts series of quasi-one-dimensional organic conductors, but also the pnictide and cuprate superconductors where several common features are observed.
\end{abstract}

PACS numbers: 71.10.Hf,71.10.Li,74.70.Kn

\section{INTRODUCTION}

The metallic state of unconventional superconductors reveals most often anomalies when superconductivity takes place in the vicinity of a density-wave instability. This is particularly exemplified in the (TMTSF) ${ }_{2} X$ series of quasi-one-dimensional organic conductors, the so-called Bechgaard salts, which develop superconductivity (SC) on the verge of a spin-density-wave (SDW) state under pressure ${ }^{1 / 5}$. Anomalous enhancement of the NMR spin-lattice relaxation rate, $T_{1}^{-1},{ }^{416} 99$ by SDW fluctuations, and linear temperature resistivity, 10 12 stand out as among the most distinctive features shown by the metallic phase of the Bechgaard salts over a broad range of pressure. Even more striking is the apparent correlation these anomalies display with the size of the SC critical temperature, $T_{c}$.

In the case of electrical transport, the correlation of $T_{c}$ with the linear- $T$ resistivity term shows how quasiparticle scattering on low energy SDW fluctuations is related to the strength of Cooper pairing and hence to the mechanism causing superconductivity $11-13$. This parallel change exhibited over the whole pressure range where $T_{c}$ is finite, contrasts with the usual picture associated with a quantum critical point. In the conventional scheme, 14 17] nonFermi-liquid (NFL) features are expected to be confined to a fan-shape region of the phase diagram, which would here emerge from the zero tem- perature SDW critical point if superconductivity was absent. Anomalies of the normal phase are actually found on a much broader range of pressure and temperature, defining an extended quantum critical domain that is intimately connected to the incursion of Cooper pairing in the phase diagram.

In this work we address this problem by examining the single particle properties of the normal state underlying the SDW-SC sequence of instabilities. The analysis is carried out by the weak coupling renormalization group method (RG) in the framework of the quasi-1D electron gas model $\frac{18}{20}$ which is generic of the close proximity of antiferromagnetism to superconductivity in lowdimensional compounds like the Bechgaard salts.

Previous one-loop RG calculations carried out along these lines have shown how the interplay between antiferromagnetism and superconductivity relies on the interdependent pairings of electron-electron (Cooper) and electron-hole (density-wave or Peierls) scattering channels, an interdependence that is present to every order in perturbation theory $\frac{18}{20}$. For repulsive intrachain interactions, the relative stability of SDW and d-wave SC (SCd) orders can thus be followed and the essence of instability lines defining the phase boundaries captured as a function of nesting deviations of the Fermi surface (FS). These deviations are commonly parameterized by the next-to-nearest neighbors transverse hopping, $t_{\perp}^{\prime}$, which modulates the warping of the FS; its amplitude, thought 
much smaller than the nearest-neighbor interchain hopping term $t_{\perp}(\sim 200 \mathrm{~K})$, acts as a low energy scale that tunes nesting frustration and simulates the primary effect of pressure 1811921222 . By raising $t_{\perp}^{\prime}$, the SDW instability line, instead of touching zero temperature at a critical $t_{\perp}^{\prime *}$, terminates where the SCd line begins at a finite $T_{c}$. It follows that a conventional pressure driven SDW quantum critical point is in a way avoided by the presence of superconductivity whose critical temperature $T_{c}$ undergoes a steady decline as $t_{\perp}^{\prime}$ increases away from $t_{\perp}^{\prime * 18 \mid 19}$.

The RG analysis has further shown that the impact of interfering pairings is not limited to the sequence of instability lines, but stretches out deeply in the metallic state ${ }^{20}$. This takes on particular importance in the superconducting sector of the calculated phase diagram. A sizable region of the normal phase can thus be defined where SDW correlations are reinforced by d-wave Cooper pairing, under the guise of a Curie-Weiss (CW) temperature variation of the SDW susceptibility above $T_{c}$. In contrast to the customary description of quantum critical phenomena, ${ }^{17}$ the enhancement is found to persist down to the lowest temperature with a strength that correlates with the size of the superconducting $T_{c}$. It has been shown how this mechanism of enhancement supplies a coherent explanation of the $\mathrm{CW}$ temperature dependence of $\left(T_{1} T\right)^{-1} \stackrel{20}{, 20}$ formerly observed in the low temperature metallic phase of different members of the Bechgaard salts series under pressure. $\frac{4618|9| 23 \mid 24}{}$

In what follows, we pursue the above program a step further and study for the same model conditions the single particle properties of the normal state. This is achieved by using the one-loop results for the scattering amplitudes in the two-loop calculation of the one-particle Matsubara self-energy, $\Sigma\left(\mathbf{k}_{F}, i \omega_{\nu}\right)$, on the FS. The calculation is carried out by the RG method over the whole temperature interval of the normal state, that is from the high temperature - $1 \mathrm{D}$ - domain down to arbitrarily far below the deconfinement temperature $T_{X}$ for normal electrons. In lowest order ${ }^{24 \mid 25}, T_{X} \sim t_{\perp}$, which fixes the temperature scale under which transverse motion of electrons becomes quantum mechanically coherent and the electron system crosses over to a two-dimensional behavior. In the case of weakly dimerized chains and relatively weak interactions relevant to the Bechgaard salts, this corresponds to the broad, but rather unexplored temperature region, ${ }^{26}$ where anomalies of the normal state can occur and instabilities of the metallic state against longrange order take place, these becoming ultimately affected by the presence of the much smaller scale $t_{\perp}^{\prime} \ll t_{\perp}$.

The momentum resolved quasi-particle weight $z\left(\mathbf{k}_{F}\right)$ on the FS along with the renormalization factors for the perpendicular hopping amplitudes can thus be extracted from the RG flow of $\Sigma\left(\mathbf{k}_{F}, i \omega_{\nu}\right)$. In the low temperature region where the normal phase is subjected to instabilities of the electron gas, the present analysis demarcates from previous works based on mean-field $\sqrt{27-29}$ and infinite transverse dimensions treatments of $t_{\perp}, \sqrt{30} 33$ in which the momentum dependent details of the FS warping are con- sidered as virtually irrelevant and the possibility of instabilities of the electron gas, absent, in weak coupling. In the high temperature region above $T_{X}$, namely where the influence of $t_{\perp}$ is perturbative, the results show some concordance with these earlier investigations.

Our analysis is completed by performing from the Padé approximants method, the analytic continuation of $\Sigma\left(\mathbf{k}_{F}, i \omega_{\nu}\right)$ to its retarded form, $\Sigma^{\mathrm{R}}\left(\mathbf{k}_{F}, \omega\right)$, defined on the real frequency axis $\omega$. We evaluate one-particle spectral quantities on the FS. As a function of energy and temperature, we examine deviations from the Fermi liquid predictions as $t_{\perp}^{\prime}$ tunes the SDW-SCd sequence of instabilities in the phase diagram.

A particular emphasis is put on the momentum resolved electron-electron scattering rate, $\tau_{\mathbf{k}_{F}}^{-1}$, on the FS, a key ingredient entering in resistivity when Umklapp scattering is present. The superconducting region of the phase diagram discloses an anomalous behavior of $\tau_{\mathbf{k}_{F}}^{-1}$, well captured by the superimposition of a linear- $T$ and $T^{2}$ temperature dependences within the $\mathrm{CW}$ domain of spin fluctuations. The decay of the NFL linear- $T$ component alongside the emergence of a regular quadratic contribution scale with the evolution of $T_{c}$ over a wide range of $t_{\perp}^{\prime}$, in accordance with an expanded region of quantum criticality for spin fluctuations. The $\tau_{\mathbf{k}_{F}}^{-1}$ anisotropy around the FS is a fingerprint of Cooper pairing that modifies the scattering of quasi-particles by spin fluctuations in the normal state.

In Sec.II, the quasi-1D electron gas model is introduced, followed in Sec. III by the calculation of oneparticle Matsubara self-energy on the Fermi surface from the one-loop scattering amplitudes. In Sec. IV, we proceed with the calculation of quasi-particle weight and renormalization of the transverse hopping integrals. By means of analytical continuation, we examine different spectral properties of the normal state quasi-particles for the quasi-1D electron gas model. In Sec. V, we discuss the results in connection with experiments in systems like the Bechgaard salts and conclude.

\section{MODEL}

We consider the quasi-1D electron gas model for a linear array of $N_{\perp}$ conducting chains of length $L$. The partition function of the model can be expressed as a functional integral

$$
Z=\iint \mathfrak{D} \psi^{*} \mathfrak{D} \psi e^{S_{0}\left[\psi^{*}, \psi\right]+S_{I}\left[\psi^{*}, \psi\right]},
$$

over the set of anticommuting fields $\left\{\psi_{p, \sigma}^{(*)}(\bar{k})\right\}$ for right $(p=+)$ and left $(p=-)$ moving electrons of spin $\sigma$ and momentum-frequency vector $\bar{k}=\left(\mathbf{k}, i \omega_{\nu}\right)$. Here $\mathbf{k}=$ $\left(k, k_{\perp}\right)$ and $\omega_{\nu}=(2 \nu+1) \pi T$ are the wave vector and the fermion Matsubara frequency, respectively $\left(\hbar=1, k_{B}=\right.$ 1 , throughout). The free part, $S_{0}$, of the action is given 
by

$$
S_{0}\left[\psi^{*}, \psi\right]=\sum_{p, \bar{k}, \sigma}\left[G_{p}^{0}(\bar{k})\right]^{-1} \psi_{p, \sigma}^{*}(\bar{k}) \psi_{p, \sigma}(\bar{k})
$$

where

$$
G_{p}^{0}(\bar{k})=\left[i \omega_{\nu}-E_{p}(\mathbf{k})\right]^{-1},
$$

is the one-electron bare propagator. The energy spectrum of the model takes the form

$$
E_{p}(\mathbf{k})=v_{F}\left(p k-k_{F}\right)-2 t_{\perp} \cos k_{\perp}-2 t_{\perp}^{\prime} \cos 2 k_{\perp},
$$

where $v_{F}$ and $k_{F}=\pi / 2$ are the longitudinal Fermi velocity and wave vector; $t_{\perp}$ is the nearest-neighbor hopping integral in the perpendicular direction (the lattice constants are set to unity). The small transverse second nearest-neighbor hopping $t_{\perp}^{\prime} \ll t_{\perp}$ paramaterizes deviations to perfect nesting of the open Fermi surface, which simulates the most important effect of the pressure in our model. The parameters of the quasi-1D spectrum are fixed in accordance to the range found in band calculations for the Bechgaard salts, that is to say with a longitudinal Fermi energy $E_{F} \simeq 15 t_{\perp} \simeq 3000 \mathrm{~K}$, which is an order of magnitude larger than $t_{\perp}{ }^{34}$ 36; the bandwidth cutoff $E_{0}=2 E_{F}\left(\equiv \pi v_{F}\right)$, is fixed as twice the longitudinal Fermi energy.

The interacting part, $S_{I}$, of the action takes the form

$$
\begin{aligned}
S_{I}=-\frac{\pi v_{F}}{T L N_{\perp}} \sum_{\{\bar{k}, \sigma\}}\left[g_{\{\sigma\}}\left(k_{\perp 1}^{\prime}, k_{\perp 2}^{\prime} ; k_{\perp 1}, k_{\perp 2}\right) \psi_{+, \sigma_{1}^{\prime}}^{*}\left(\bar{k}_{1}^{\prime}\right) \psi_{-, \sigma_{2}^{\prime}}^{*}\left(\bar{k}_{2}^{\prime}\right) \psi_{-, \sigma_{2}}\left(\bar{k}_{2}\right) \psi_{+, \sigma_{1}}\left(\bar{k}_{1}\right)\right. \\
+\frac{1}{2} g_{3}\left(k_{\perp 1}^{\prime}, k_{\perp 2}^{\prime} ; k_{\perp 1}, k_{\perp 2}\right) \psi_{+, \sigma_{1}}^{*}\left(\bar{k}_{1}^{\prime}\right) \psi_{+, \sigma_{2}}^{*}\left(\bar{k}_{2}^{\prime}\right) \psi_{-, \sigma_{2}}\left(\bar{k}_{2}\right) \psi_{-, \sigma_{1}}\left(\bar{k}_{1}\right) \\
\left.+\frac{1}{2} g_{3}\left(k_{\perp 1}^{\prime}, k_{\perp 2}^{\prime} ; k_{\perp 1}, k_{\perp 2}\right) \psi_{-, \sigma_{1}}^{*}\left(\bar{k}_{1}^{\prime}\right) \psi_{-, \sigma_{2}}^{*}\left(\bar{k}_{2}^{\prime}\right) \psi_{+, \sigma_{2}}\left(\bar{k}_{2}\right) \psi_{+, \sigma_{1}}\left(\bar{k}_{1}\right)\right] \delta_{\mathbf{k}_{1}+\mathbf{k}_{2}=\mathbf{k}_{1}^{\prime}+\mathbf{k}_{2}^{\prime}( \pm \mathbf{G})},
\end{aligned}
$$

which comprises a normal part

$$
g_{\{\sigma\}}\left(k_{\perp 1}^{\prime}, k_{\perp 2}^{\prime} ; k_{\perp 1}, k_{\perp 2}\right)=g_{2} \delta_{\sigma_{2} \sigma_{2}^{\prime}} \delta_{\sigma_{1} \sigma_{1}^{\prime}}-g_{1} \delta_{\sigma_{1}^{\prime} \sigma_{2}} \delta_{\sigma_{2}^{\prime} \sigma_{1}},
$$

regrouping the bare backward $\left(g_{1}\right)$ and forward $\left(g_{2}\right)$ scattering amplitudes between right and left moving electrons. The half-filling character of the band - due to the small dimerization gap $\Delta_{D}$ of the chains - gives rise to Umklapp scattering $\stackrel{37}{ }$, of bare amplitude

$$
g_{3}\left(k_{\perp 1}^{\prime}, k_{\perp 2}^{\prime} ; k_{\perp 1}, k_{\perp 2}\right) \approx g_{1} \Delta_{D} / E_{F},
$$

and for which momentum conservation is satisfied by adding the longitudinal reciprocal lattice vector $\bar{G}=$ $(\mathbf{G}, 0)$, where $\mathbf{G}=\left(4 k_{F}, 0\right)$. All the bare - initial - couplings are normalized by the bandwidth $\pi v_{F}$. They are local or intrachain like and independent of the transverse momentum, the momentum dependence being generated by the RG flow.

Initial couplings can be seen as input phenomenological parameters of an effective theory of the electron gas. Following the example of Fermi liquid theory, their values can be delimited for the most part by experiments. The temperature dependent enhancement of uniform magnetic susceptibility, for example, is known to be related to the backscattering amplitude ${ }^{38 / 39}$; its observation is compatible with the range of values $g_{1} \approx 0.2-0.4^{7}$. The weak dimerization of organic stacks observed by X-rays 40 leads, according to band calculations, to the small ratio $\Delta_{D} / E_{F} \sim 0.1$ for the dimerization gap, $\frac{41}{,}$ which according to 77 yields $g_{3} \approx 0.02-0.04$. As for the range of $g_{2}$, it can be fixed by matching the temperature scale of the SDW instability in low pressure experiments, ${ }^{2142}$ namely $T_{\mathrm{SDW}} \approx 10-25 \mathrm{~K}$, to the predictions of RG calculations for $T_{\mathrm{SDW}}$ at small $t_{\perp}^{\prime}$; one thus finds $g_{2} \approx 0.5-0.7$. Although the above set of figures is merely indicative of what may occur in practice, it turns out to be rather generic of the succession of ground states that actually takes place in low dimensional materials like the Bechgaard salts.

In the following, we shall use $g_{1}=g_{2} / 2=0.32$ and $g_{3}=0.02$ as initial couplings. There is nothing special about this choice other than it coincides with the one used in previous RG analysis for the phase diagram and NMR $T_{1}^{-118 \mid 20}$, thus allowing a common framework for the connection with experiments.

\section{RENORMALIZATION GROUP ANALYSIS}

We apply a Kadanoff-Wilson RG approach to the partition function $Z^{43 \mid 44}$. In the present quasi-1D problem, this is achieved by first dividing the open Fermi surface (FS) into 32 pieces or patche $\$ 4556$, each centered on a particular transverse momentum $k_{\perp}$ that parametrizes a point $\mathbf{k}_{F, p}=\left(k_{F, p}\left(k_{\perp}\right), k_{\perp}\right)$ on the FS sheet, $p= \pm$ (Fig. 1), where the longitudinal component $k_{F, p}\left(k_{\perp}\right)$ follows from the FS equation, $E_{p}\left(\mathbf{k}_{F, p}\right)=0$.

The RG proceeds from successive partial traces of $Z$ over a subset of variables $\bar{\psi}^{(*)}$ of the action in an energy 


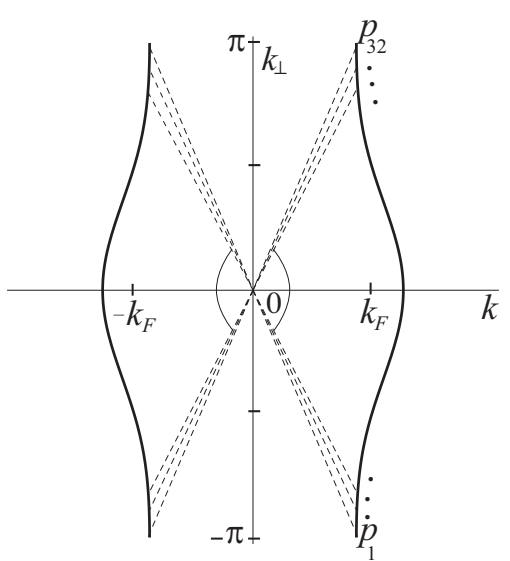

FIG. 1: The Fermi surface of the quasi-one dimensional electron gas model. Each dashed line ends to a patch $p_{i=1 \ldots 32}$ on each Fermi sheet.

shell of thickness $\delta E_{0}(\ell) / 2=E_{0}(\ell) d \ell / 2$, above and below the FS. This is done in a perturbative scheme in the energy intervals $\Delta_{ \pm}=\left[ \pm \frac{1}{2} E_{0}(\ell), \pm E_{F}\right]$, ranging from the cutoff energy $E_{F}$ down to $\frac{1}{2} E_{0}(\ell)=\frac{1}{2} E_{0} e^{-\ell}$, above and below the FS at the step $\ell \geq 0$. For a warped Fermi surface like in Fig. 1. the RG integration proceeds with a cascade of contractions of three-particles diagrams over the whole energy intervals $\Delta_{ \pm}$. A momentum dependence for the scattering amplitudes is generated, which is restricted to the set $\left\{k_{\perp}\right\}$ of three transverse external variables at the energy distance $\frac{1}{2} E_{0}(\ell)$ from the FS. At the one-loop level for instance 47 , the procedure is equivalent to the one-particle irreducible scheme of the functional $\mathrm{RG}^{48}$, as previously applied to the model under study in Ref $\frac{18}{18}$.

\section{A. Summary of one-loop results}

Before embarking on the study of one-particle properties, it is useful to first single out the main results of the previous RG calculations carried out at the one-loop level, 1820 These refer to the particle and hole excitations entering in the single particle Matsubara self-energy at the two-loop level.

At the one-loop level the cascade of shell contractions, $\mathcal{O}\left(S_{I}^{2}\right)$, in interactions yields the flow equations shown diagrammatically in Fig. 2 2 a for the normal and Umklapp scattering amplitudes. The related expressions were obtained by Nickel et al! ${ }^{18}$ [Eqs. (10)-(12) of Ref. $\left.{ }^{[18}\right]$, and are summarized as follows:

$$
\begin{aligned}
\partial_{\ell} g_{i=1,2}\left(k_{\perp 1}^{\prime}, k_{\perp 2}^{\prime} ; k_{\perp 1}, k_{\perp 2}\right) & =\sum_{\mathcal{P}_{n n^{\prime}}}\left\{\epsilon_{C, i}^{n, n^{\prime}}\left\langle g_{n} \cdot g_{n^{\prime}} \cdot \partial_{\ell} \mathcal{L}_{C}\right\rangle_{k_{\perp}}\right. \\
& \left.+\epsilon_{P, i}^{n, n^{\prime}}\left\langle g_{n} \cdot g_{n^{\prime}} \cdot \partial_{\ell} \mathcal{L}_{P}\right\rangle_{k_{\perp}}\right\}, \\
\partial_{\ell} g_{3}\left(k_{\perp 1}^{\prime}, k_{\perp 2}^{\prime} ; k_{\perp 1}, k_{\perp 2}\right)= & \sum_{\mathcal{P}_{3 n}} \epsilon_{P, 3}^{3, n}\left\langle\tilde{g}_{3} \cdot \tilde{g}_{n} \cdot \partial_{\ell} \mathcal{L}_{P}\right\rangle_{k_{\perp}},(8)
\end{aligned}
$$

(a) $\partial_{\ell}$
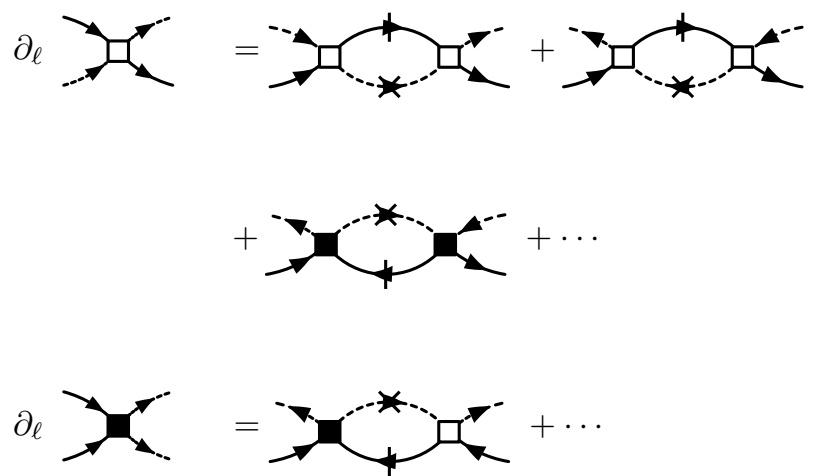

(b)

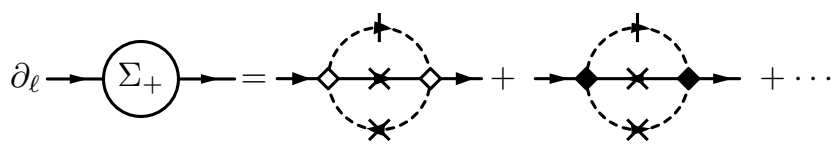

FIG. 2: Flow equations for : (a) the $g_{1,2}$ (open square) and Umklapp $g_{3}$ (full square) scattering amplitudes for right (continuous line) and left (dashed line) moving electrons; (b) the one-particle Matsubara self-energy, $\Sigma_{+}$. The crossed and slashed lines refer to the high energy intervals $\Delta_{ \pm}$and the last outer energy shell, respectively (permutations between crossed and slashed line are not shown).

where the $k_{\perp}$-dependence of convolution products, constrained by momentum conservation, has been masked for simplicity; here $\langle\ldots\rangle_{k_{\perp}}$ stands for an average over $k_{\perp}$. The Peierls and Cooper loops at finite $T$ read $\frac{18}{}$

$$
\begin{aligned}
& \partial_{\ell} \mathcal{L}_{P, C}\left(k_{\perp}, q_{P, C}\right)=\sum_{\nu= \pm} \theta\left(\left|E_{0}(\ell) / 2+\nu \mathcal{A}_{P, C}\right|-E_{0}(\ell) / 2\right) \\
& \times \frac{1}{2}\left(\tanh \frac{E_{0}(\ell)+2 \nu \mathcal{A}_{P, C}}{4 T}+\tanh \frac{E_{0}(\ell)}{4 T}\right) \\
& \times \frac{E_{0}(\ell)}{4 E_{0}(\ell)+2 \nu \mathcal{A}_{C, P}}
\end{aligned}
$$

which are evaluated at $q_{P}=k_{\perp 1}-k_{\perp 1}^{\prime}$ and $q_{C}=$ $k_{\perp 1}+k_{\perp 2}$, respectively; $\mathcal{A}_{P, C}\left(k_{\perp}, q_{P, C}\right)=-E_{+}\left(k_{F}, k_{\perp}\right) \mp$ $E_{-}\left(-k_{F}, k_{\perp}+q_{P, C}\right)$ and $\theta(x)$ is the step function. For a given $g_{i}$, the related sum in (8) collect all possibilities $\left\{\mathcal{P}_{n n^{\prime}}\right\}$ of diagrams belonging to the Peierls and Cooper scattering channels $P$ and $C$. The coefficients $\epsilon_{C, P, i}^{n n^{\prime}}$ fix the sign and spin multiplicity of each loop contribution; it differs for closed loops $\left(\epsilon_{P, i}^{n n^{\prime}}=-2\right)$, vertex corrections $\left(\epsilon_{P, i}^{n n^{\prime}}=1\right)$ and ladder $\left(\epsilon_{C, i}^{n n^{\prime}}=-\epsilon_{P, i}^{n n^{\prime}}=-1\right)$ diagrams.

The equation for the normal part $g_{\sigma}\left(g_{1}, g_{2}\right)$ superimposes Peierls and Cooper loops that will mix and interfere at every order following the $\ell$-integration. As for the Umklapp amplitude, $g_{3}$, it only contains Peierls loops, but in accordance with (8), connects in a key manner with Cooper pairing via a linear coupling to normal interactions (Fig. 2).

The integration of RG equations is conducted up to $\ell \rightarrow \infty$ yielding the scattering amplitudes at temperature $T$, which stands as a distinct parameter from $E_{0}(\ell)$. 
A singularity in the scattering amplitudes signals an instability of the metallic phase toward the formation of a collective state. Strictly speaking, for an effective twodimensional electron gas, fluctuations would prevent the possibility of long-range order at finite $T$. Potential couplings or/and single particle hopping in a third direction are therefore needed so that a true phase transition at finite temperature can occur. The one-loop transition temperature can be seen as a characteristic scale for the onset of short-range order in two dimensions, but enough representative of the actual transition if three-dimensional effects were included.

As to the precise nature of the normal state instability, it is best provided from the most singular response function $\chi_{\mu}$, at the 'ordering' temperature $T_{\mu}$ of the vertex part (8). In the repulsive sector of bare intrachain couplings, only the SDW and SCd susceptibilities display singularities depending on the strength of the antinesting parameter $\left.t_{\perp}^{\prime} \cdot 18\right]$

In the RG framework, the expression for the normalized susceptibility, $\tilde{\chi}_{\mu} \equiv \pi v_{F} \chi_{\mu}$, is given by

$$
\tilde{\chi}_{\mu}\left(\mathbf{q}_{\mu, 0}\right)=2 \int_{\ell}\left\langle f_{\mu}\left(k_{\perp}\right) z_{\mu}^{2}\left(k_{\perp}\right) \partial_{\ell} \mathcal{L}_{\mu}\right\rangle_{k_{\perp}} d \ell
$$

whose $k_{\perp}$ average expression depends on the vertex renormalisation factors $z_{\mu}\left(k_{\perp}\right)$ and the form factors $f_{\mathrm{SDW}}=1$ and $f_{\mathrm{SCd}}=2 \cos ^{2} k_{\perp}$ for the SDW and SCd

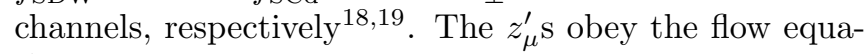
tion

$$
\partial_{\ell} z_{\mu}\left(k_{\perp}\right)=\left\langle\left(\partial_{\ell} \mathcal{L}_{\mu}\right) g_{\mu} z_{\mu}\left(k_{\perp}^{\prime}\right)\right\rangle_{k_{\perp}^{\prime}}
$$

governed by the combinations of momentum dependent couplings, $g_{\mathrm{SDW}}=g_{2}\left(k_{\perp}, k_{\perp}^{\prime} ; k_{\perp}+\right.$ $\left.\pi, k_{\perp}^{\prime}+\pi\right)+g_{3}\left(k_{\perp}^{\prime}, k_{\perp} ; k_{\perp}^{\prime}+\pi, k_{\perp}+\pi\right)$ and $g_{\mathrm{SCd}}=-g_{1}\left(k_{\perp}^{\prime},-k_{\perp}^{\prime} ;-k_{\perp}, k_{\perp}\right)-g_{2}\left(k_{\perp}^{\prime},-k_{\perp}^{\prime} ; k_{\perp},-k_{\perp}\right)$. A singularity in $g_{\mathrm{SDW}}$ (resp., $g_{\mathrm{SCd}}$ ) gives rise to a singularity in $\tilde{\chi}_{\mathrm{SDW}}\left(\right.$ resp., $\left.\tilde{\chi}_{\mathrm{SCd}}\right)$ at the ordering temperature $T_{\mathrm{SDW}}\left(\right.$ resp., $T_{c}$ ).

Thus at small $t_{\perp}^{\prime}$, nesting is sufficiently good so that a SDW instability occurs. It is found with a divergence of the static SDW susceptibility, $\tilde{\chi}_{\mathrm{SDW}}\left(\mathbf{q}_{0}\right)$,s at the temperature $T_{\mathrm{SDW}}$, when evaluated at the nesting vector $\mathbf{q}_{0}=\left(2 k_{F}, \pi\right)$ of $E_{p}(\mathbf{k})$ in the absence of $t_{\perp}^{\prime}$ (Fig. 4 . It is worth stressing here that this by no means indicates that the Cooper pairing channel is ineffective. On the contrary, the presence of electron-electron scattering and its interference with electron-hole processes reduces considerably the temperature scale $T_{\mathrm{SDW}}$ for the magnetic instability. For the parameters used here, for instance the $T_{\text {SDW }}$ obtained is reduced by a factor six or so compared to when the Peierls channel is singled out by putting to zero the Cooper loops in (8).

The signature of staggered density-wave correlations in the SDW sector of the phase diagram is also manifest in the momentum dependence of the coupling constants. The Figure 3 shows typical momentum contour map of the normal combination

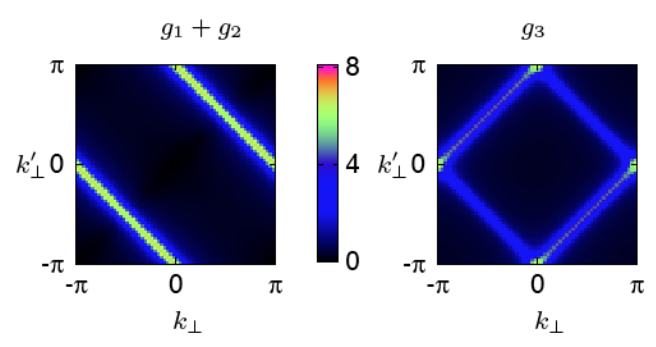

FIG. 3: (Color on line) Momentum dependence of the projected coupling constants $g_{1}\left(k_{\perp}^{\prime},-k_{\perp}^{\prime} ;-k_{\perp}, k_{\perp}\right)+$ $g_{2}\left(k_{\perp}^{\prime},-k_{\perp}^{\prime} ; k_{\perp},-k_{\perp}\right)$ and $g_{3}\left(k_{\perp}^{\prime},-k_{\perp}^{\prime} ; k_{\perp},-k_{\perp}\right)$ in the $\left(k_{\perp}, k_{\perp}^{\prime}\right)$ plane for the normal phase $(T=25 \mathrm{~K})$ of the SDW instability $\left(t_{\perp}^{\prime}=25 \mathrm{~K}\right)$.

$g_{1}\left(k_{\perp}^{\prime},-k_{\perp}^{\prime} ;-k_{\perp}, k_{\perp}\right)+g_{2}\left(k_{\perp}^{\prime},-k_{\perp}^{\prime} ; k_{\perp},-k_{\perp}\right)$, and Umklapp $g_{3}\left(k_{\perp}^{\prime},-k_{\perp}^{\prime} ; k_{\perp},-k_{\perp}\right)$, as projected in the $\left(k_{\perp}, k_{\perp}^{\prime}\right)$ plane at zero incoming pair momentum. In the normal phase $(T=25 \mathrm{~K})$ of the SDW instability at $t_{\perp}^{\prime}=25 \mathrm{~K}$, the amplitude of both $g_{1}+g_{2}$ and $g_{3}$ concentrates essentially along the lines $k_{\perp}^{\prime}=k_{\perp} \pm \pi$, namely at $\pi$ momentum transfer for staggered correlations along the transverse direction. Along the FS, as a function $k_{\perp}$, a maximum of scattering intensity for $g_{1}+g_{2}$, though tiny, is found at the best nesting points $k_{\perp}= \pm \pi / 4$ and $\pm 3 \pi / 4$, whereas for $g_{3}$, the maxima are at $k_{\perp}=0$ and edges $\pm \pi$ of the FS.

Now as $t_{\perp}^{\prime}$ is tuned to larger values, nesting further deteriorates which gradually suppresses the Peierls singularity of the density-wave channel. As shown in Fig. 44a, $T_{\text {SDW }}$ then first decreases monotonically until the critical value $t_{\perp}^{* *}(\simeq 25.6 \mathrm{~K}$ for the parameters of the model $)$ is approached where it sharply drops (Fig. 4). However, in its fall, $T_{\mathrm{SDW}}$ does not reach zero temperature, but ends at the maximum $T_{c}$ of the SCd instability line $\left(T_{c}^{*} \simeq 1.4 \mathrm{~K}\right.$ at $\left.t_{\perp}^{\prime *}\right)$, along which the static d-wave susceptibility $\tilde{\chi}_{\mathrm{SCd}}(0)$ is singular at zero Cooper pair momentum. Here the influence of SDW correlations passes to the Cooper channel by interference and leads to d-wave pairing between electrons of opposite spins on neighboring chains $49 \sqrt[491]{51}$

In the superconducting sector of the phase diagram where $t_{\perp}^{\prime} \geq t_{\perp}^{\prime *}$, SDW fluctuations display a characteristic change of regime in the normal phase. This is apparent in the $T$ variation of $\tilde{\chi}_{\mathrm{SDW}}^{-1}$ of Fig. $4 \mathrm{~b}$, where the temperature, $T_{\mathrm{CW}} \sim 10 \mathrm{~K}$, marks out the scale below which $\tilde{\chi}_{\mathrm{SDW}}$ is no longer singular, but evolves toward a finite intercept as $T \rightarrow 0$. In the scaling language, $T_{\mathrm{CW}}$ corresponds to the temperature range where the RG flow is no longer under the control of the SDW fixed point as a result of nesting deviations, but crosses over to that of the SCd instability. ${ }^{20}$ Moreover, below $T_{\mathrm{CW}}, \tilde{\chi}_{\mathrm{SDW}}^{-1}$ evolves towards the $T \rightarrow 0$ intercept with a finite positive slope, implying that $\tilde{\chi}_{\mathrm{SDW}}\left(\mathbf{q}_{0}\right)$ is still enhanced. The SDW enhancement, though non singular, is well captured by the 


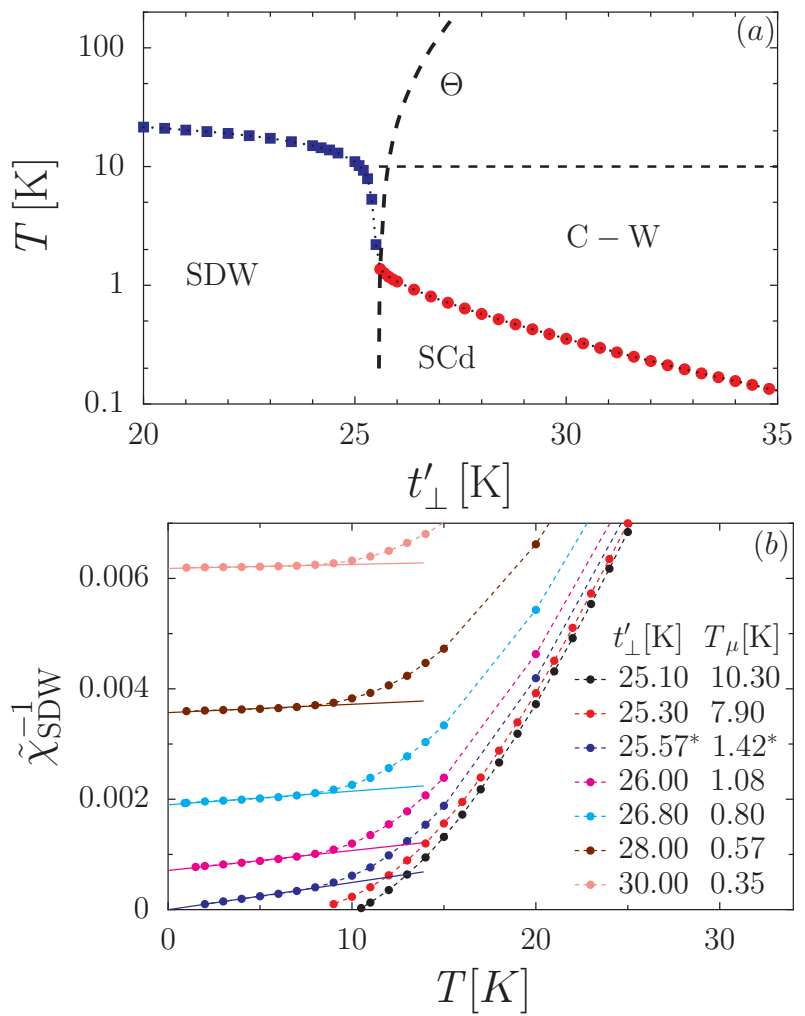

FIG. 4: (Color on line) (a): Calculated phase diagram of the quasi-one-dimensional electron gas model from the renormalization group method at the one-loop level; $T_{\mathrm{CW}}$ and $\Theta$ define the temperature region and of the $\mathrm{CW}$ behavior for the inverse normalized SDW response function shown in (b) for different $t_{\perp}^{\prime}$.

Curie-Weiss expression,

$$
\tilde{\chi}_{\mathrm{SDW}}\left(\mathbf{q}_{0}\right) \simeq \frac{C}{T+\Theta}, \quad\left(T<T_{\mathrm{CW}}\right),
$$

where $C, \Theta>0$ (Fig. 4 b). It follows that the SDW correlation length, $\xi \sim(T+\Theta)^{-1 / 2}$, shows a regular reinforcement down to the lowest temperature allowed by the calculations, namely $T_{c}$.

Interestingly enough, the CW enhancement turns out to be absent when the Cooper channel is switched off and all the Cooper loops in (8) or Fig. 2-a are put equal to zero. In the absence of Cooper pairing, the slope of $\tilde{\chi}_{\mathrm{SDW}}^{-1}$ is vanishingly small and $\tilde{\chi}_{\mathrm{SDW}}^{-1}$ is essentially constant below $T_{\mathrm{CW}}$ at all $t_{\perp}^{\prime} \geq t_{\perp}^{\prime *}$. According to Fig. 4 $\mathrm{b}$ when Cooper pairing is included, it is only when $t_{\perp}^{\prime}$ is sufficiently far from $t_{\perp}^{\prime *}$ or $T_{c}$ small enough compared to $T_{c}^{*}$ that the slope $1 / C$ tends to that of the single channel limit. The origin of CW SDW enhancement is a direct consequence of the positive feedback of the Cooper (dwave ) pairing channel on the Peierls one through the linear coupling to Umklapp scattering [Eqs. [8]].

Reinforcement of SDW correlations by Cooper pairing in the CW domain can also be seen in the temperature dependent intensity of scattering amplitudes in momentum space. In Fig. 5, the low temperature evolution of
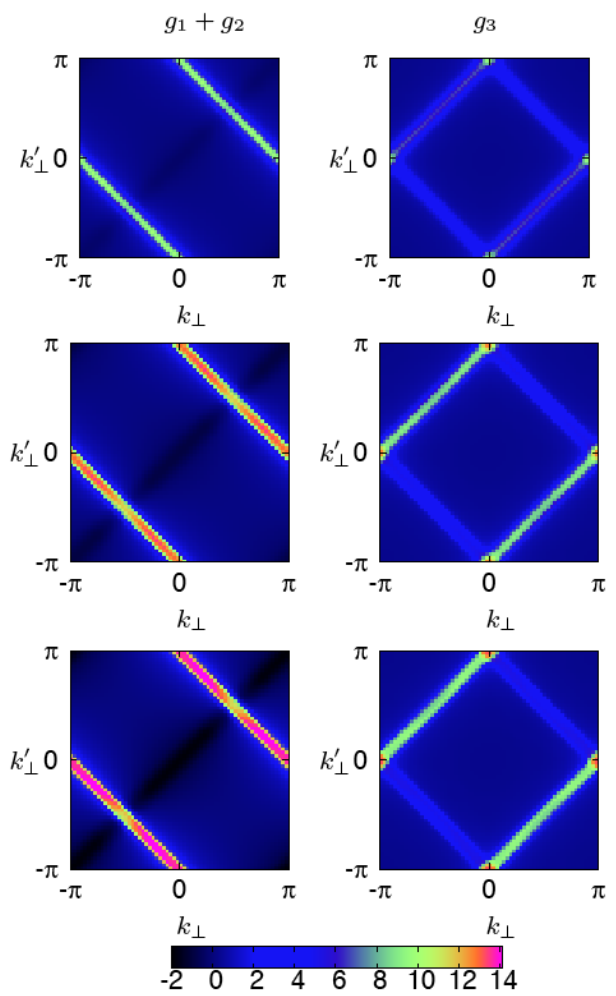

FIG. 5: (Color on line) Low temperature momentum dependence of the coupling constants $g_{1}\left(k_{\perp}^{\prime},-k_{\perp}^{\prime} ;-k_{\perp}, k_{\perp}\right)+$ $g_{2}\left(k_{\perp}^{\prime},-k_{\perp}^{\prime} ; k_{\perp},-k_{\perp}\right)$ (left) and $g_{3}\left(k_{\perp}^{\prime},-k_{\perp}^{\prime} ; k_{\perp},-k_{\perp}\right)$ (right) in the $\left(k_{\perp}, k_{\perp}^{\prime}\right)$ plane for the normal phase of the SCd instability $\left(t_{\perp}^{\prime}=29 \mathrm{~K}\right) ; T=15 \mathrm{~K}$ (top), $8 \mathrm{~K}$ (middle) and $4 \mathrm{~K}$ (bottom).

$g_{1}+g_{2}$ and $g_{3}$ is shown above and below $T_{\mathrm{CW}}$ in the $\mathrm{SCd}$ region of the phase diagram at $t_{\perp}^{\prime}=29 \mathrm{~K}\left(T_{c}=0.4 \mathrm{~K}\right)$. Above $T_{\mathrm{CW}}$ (top of Fig. 5), the momentum profiles of $g_{1}+g_{2}$ and $g_{3}$ at zero incoming pair momentum display structures similar to those of the normal phase of the SDW instability (Fig. 3). However, following the entry in the CW domain, the scattering intensity of $g_{1}+g_{2}$ at momentum transfer $q_{\perp}= \pm \pi$ increases continuously as the temperature is lowered over the whole antinodal region of the FS at $k_{\perp} \neq \pm \pi / 2$. The increase is indicative of a regular increase of staggered density-wave correlations which is concomitant to a growing attractive component along the diagonal $k_{\perp} \sim k_{\perp}^{\prime}$. This is distinctive of constructive interference between the Peierls and Cooper scattering channels. As shown in Fig. 5 , the interference leads to an increase of Umklapp scattering at large momentum transfer, in particular for $k_{\perp} \sim 0$ and $k_{\perp} \sim \pm \pi$, which contributes to the regular increase of SDW correlations in the CW temperature domain (Fig. 4-b).

Regarding the CW scale $\Theta$, it rises from zero at $t_{\perp}^{\prime *}$ and increases rapidly as $t_{\perp}^{\prime}$ gets larger or $T_{c}$ decreases (Fig. 4 a). The rapid growth of $\Theta$ reflects the decrease of spin fluctuations amplitude as a function of $t_{\perp}^{\prime}$, as shown by the decline of zero temperature intercept of 
$\tilde{\chi}_{\mathrm{SDW}}\left(\mathbf{q}_{0}, T \rightarrow 0\right)(=C / \Theta)$ in Fig. 4 4 b. It can be identified with a characteristic energy scale for SDW fluctuations that softens and becomes critical as $t_{\perp}^{\prime *}$ is approached from above in the phase diagram. As a function of the tuning parameter $t_{\perp}^{\prime}, \Theta$ evolves indeed according the critical form

$$
\Theta \approx A\left(t_{\perp}^{\prime}-t_{\perp}^{\prime *}\right)^{\eta}
$$

which follows a linear variation, namely $\eta \simeq 1$, over the whole interval $t_{\perp}^{\prime}>t_{\perp}^{\prime *}$. Attempting a quantum scaling approach to $\eta$, one can write $\eta=\nu z$, so that linearity is compatible with the one-loop exponents $\nu=1 / 2$ for correlation length and $z=2$ for dynamics of SDW fluctuations. It has been checked that within one-loop approximation, linearity is essentially independent of the initial values of coupling constants. The slope $A$, however, is not universal: a small increase in the initial value of Umklapp term, $g_{3}$, for instance, reduces appreciably the amplitude of $A$ and then the rise of $\Theta$ as a function of $t_{\perp}^{\prime}$; this increases the $\mathrm{CW}$ slope $1 / C$ for $\tilde{\chi}_{\mathrm{SDW}}^{-1}$ and the amplitude of spin fluctuations above $T_{c}$.

\section{B. Two-loop one-particle self-energy}

The successive partial traces of $Z$ in the RG transformation yield a cascade of contractions for the $n \geq 3$ particles vertex functions that are generated at each step of procedure. For the 3-particles vertex, two cascades of contractions for two internal fermion lines, followed by a third line contraction on the final shell, yield at every step the two-loop corrections of one-particle self-energy, $\delta \Sigma_{p} 44 \mid 4752$. Details of the $\delta \Sigma_{p}$ calculation are given in Appendix A.

In the following, we shall focus on the corrections evaluated on the (bare) FS where the inverse one-particle propagator of $S_{0}$ at the step $\ell$ of the $\mathrm{RG}$, is of the form

$$
\left[G_{p}^{0}\left(\mathbf{k}_{F, p}, \bar{k}_{\perp}\right)\right]_{\ell}^{-1}=\left[z\left(\bar{k}_{\perp}\right) G_{p}^{0}\left(\mathbf{k}_{F, p}, \bar{k}_{\perp}\right)\right]^{-1}+z_{\perp}\left(\bar{k}_{\perp}\right)
$$

where $\bar{k}_{\perp}=\left(k_{\perp}, i \omega_{\nu}\right)$. In accordance with the results of Appendix A, the one-particle self-energy corrections give rise to two renormalization factors, $z\left(\bar{k}_{\perp}\right)$ and $z_{\perp}\left(\bar{k}_{\perp}\right)$, submitted to the initial conditions $z\left(\bar{k}_{\perp}\right)=1$ and $z_{\perp}\left(\bar{k}_{\perp}\right)=0$ at $\ell=0$, for all $\bar{k}_{\perp}$.

Both $z$ and $z_{\perp}$ are real quantities, which from Eq. A18 of the Appendix A, obey the following flow equations

$$
\begin{aligned}
& \partial_{\ell} \ln z\left(\bar{k}_{\perp}\right)=\frac{1}{2} \iint \frac{d k_{\perp}^{\prime}}{2 \pi} \frac{d q_{\perp}}{2 \pi} \\
& \times\left\{\left(g_{2}\left(\widetilde{k}_{\perp 1}\right) g_{1}\left(\widetilde{k}_{\perp 1}\right)-g_{2}^{2}\left(\widetilde{k}_{\perp 1}\right)-g_{1}^{2}\left(\widetilde{k}_{\perp 1}\right)\right) I_{1}\left(\widetilde{k}_{\perp}, i \omega_{\nu}\right)\right. \\
& -\left(g_{2}^{2}\left(\widetilde{k}_{\perp 2}\right)+g_{1}^{2}\left(\widetilde{k}_{\perp 2}\right)-g_{2}\left(\widetilde{k}_{\perp 2}\right) g_{1}\left(\widetilde{k}_{\perp 2}\right)\right) I_{2}\left(\widetilde{k}_{\perp}, i \omega_{\nu}\right) \\
& \left.-\left(g_{3}^{2}\left(\widetilde{k}_{\perp 3}\right)+g_{3}^{2}\left(\widetilde{k}_{\perp 4}\right)-g_{3}\left(\widetilde{k}_{\perp 3}\right) g_{3}\left(\widetilde{k}_{\perp 4}\right)\right) I_{3}\left(\widetilde{k}_{\perp}, i \omega_{\nu}\right)\right\},
\end{aligned}
$$

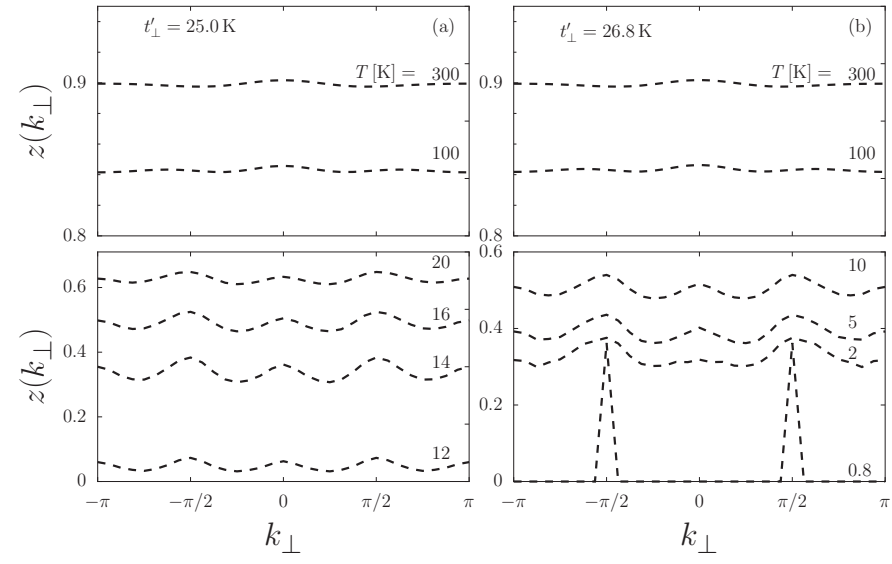

FIG. 6: Variation of the quasi-particle weight on the Fermi surface as a function of $k_{\perp}$ at different temperatures. (a): $\mathrm{SDW}\left(t_{\perp}^{\prime}=25 \mathrm{~K}<t_{\perp}^{\prime *}, T_{\mathrm{SDW}} \simeq 12 \mathrm{~K}\right) ;(\mathrm{b}): \operatorname{SCd}\left(t_{\perp}^{\prime}=\right.$ $\left.26.8 \mathrm{~K}>t_{\perp}^{\prime *}, T_{c}=0.8 \mathrm{~K}\right)$.

and

$$
\begin{aligned}
& \partial_{\ell} z_{\perp}\left(\bar{k}_{\perp}\right)=\left[z\left(\bar{k}_{\perp}\right)\right]^{-1} \frac{1}{2} \iint \frac{d k_{\perp}^{\prime}}{2 \pi} \frac{d q_{\perp}}{2 \pi} \\
& \times\left\{\left(g_{2}\left(\widetilde{k}_{\perp 1}\right) g_{1}\left(\widetilde{k}_{\perp 1}\right)-g_{2}^{2}\left(\widetilde{k}_{\perp 1}\right)-g_{1}^{2}\left(\widetilde{k}_{\perp 1}\right)\right) I_{1}^{\prime}\left(\widetilde{k}_{\perp}, i \omega_{\nu}\right)\right. \\
& -\left(g_{2}^{2}\left(\widetilde{k}_{\perp 2}\right)+g_{1}^{2}\left(\widetilde{k}_{\perp 2}\right)-g_{2}\left(\widetilde{k}_{\perp}\right) g_{1}\left(\widetilde{k}_{\perp 2}\right)\right) I_{2}^{\prime}\left(\widetilde{k}_{\perp}, i \omega_{\nu}\right) \\
& \left.-\left(g_{3}^{2}\left(\widetilde{k}_{\perp 3}\right)+g_{3}^{2}\left(\widetilde{k}_{\perp 4}\right)-g_{3}\left(\widetilde{k}_{\perp 3}\right) g_{3}\left(\widetilde{k}_{\perp 4}\right)\right) I_{3}^{\prime}\left(\widetilde{k}_{\perp}, i \omega_{\nu}\right)\right\}
\end{aligned}
$$

where $\widetilde{k}_{\perp i}$ and $\widetilde{k}_{\perp}$ are defined in $\mathrm{A} 4$ and $\mathrm{A} 7$, respectively. The temperature dependent coefficients $I_{i}$ and $I_{i}^{\prime}$ are given by A11 A12 and (A17) of Appendix A. The integration of (15 16), together with (8), is carried out up to $\ell \rightarrow \infty$, which yields the $z$ and $z_{\perp}$ factors at temperature $T$. It worth stressing that both two-loop and one-loop diagrams are calculated using free propagators (see Appendix A).

\section{RESULTS}

\section{A. Quasi-particle weight}

An important quantity entering in the description of quasi-particles and that can be extracted from the oneparticle self-energy is the 'momentum resolved' quasiparticle weight, $z\left(k_{\perp}\right) \equiv z\left(\mathbf{k}_{F, p}\left(k_{\perp}\right), i \omega_{\nu=0}\right)$, on the FS. It is obtained from the solution of Eq. 15 at $\omega_{\nu=0}=\pi T$. Typical $k_{\perp}$ and $T$ dependences of $z\left(k_{\perp}\right)$ in the SDW and SC parts of the phase diagram are shown in Fig. 6.

In the SDW region and for the 1D temperature domain, namely well above the scale of one-particle transverse coherence, $T_{X} \sim t_{\perp}, z\left(k_{\perp}\right)$ is only weakly reduced and displays little minima at $k_{\perp} \sim \pm \pi / 2$. According to (4), the $t_{\perp}$ part of the spectrum vanishes at those points. 


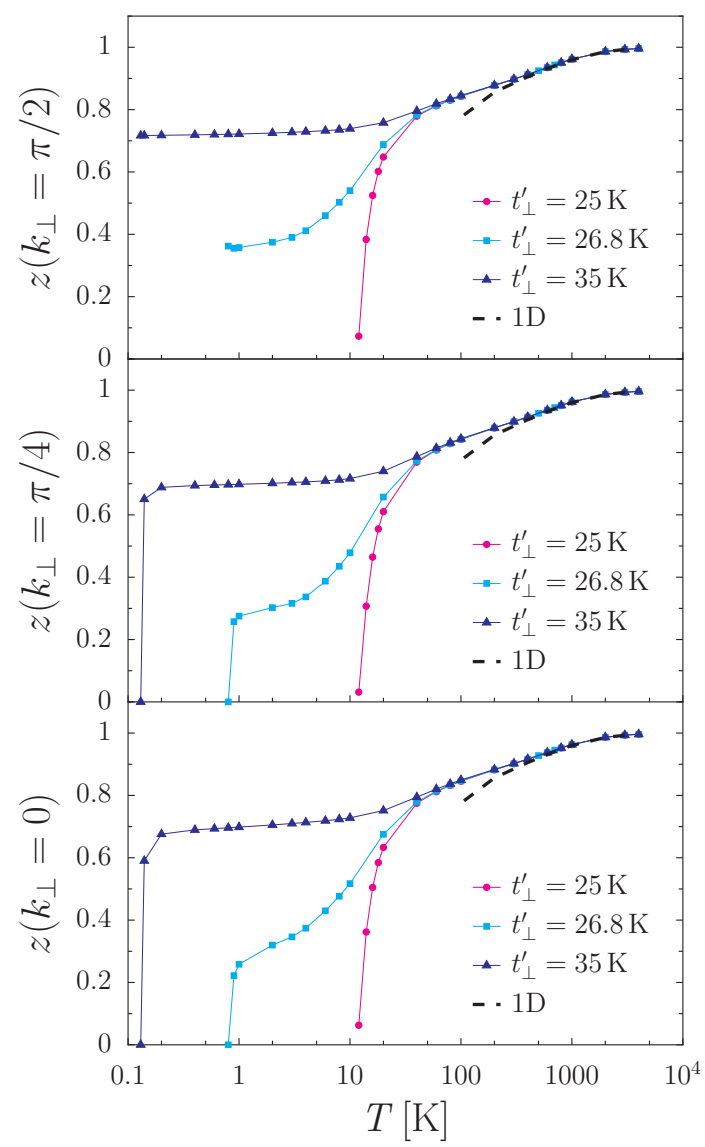

FIG. 7: (Color on line) Quasi-particle weight $z\left(k_{\perp}\right)$ as a function of temperature (log. scale) and antinesting parameter $t_{\perp}^{\prime}$ for $k_{\perp}=0, \pi / 4$, and $\pi / 2$. The dashed line corresponds to the power law decay $z_{1 \mathrm{D}}$ of the one-dimensional limit.

From a perturbation viewpoint of $t_{\perp}$, this implies that 1D effects are the strongest there. Such a high temperature modulation, thought small here, agrees with the results of earlier investigations based on perturbative and mean field treatments of $t_{\perp} 27 \sqrt[33]{3}$, which find the same location for the spectral weight minima on the FS. Fig. 7 further shows that in this $1 \mathrm{D}$ domain, $z\left(k_{\perp}\right) \sim T^{\alpha}$ and decays as a non universal power law in temperature $\left(\alpha \sim \mathcal{O}\left(g^{2}\right)\right)$, in accordance with the summation of next-to-leading logarithmic singularities for the self-energy, a well known result of the 1D electron gas model.4315354 (dashed line of Fig. 7).

As $T$ goes below $T_{X}$, the influence of the $k_{\perp}$-dependent warping of the FS becomes clearly non perturbative. The temperature decay of $z$, thought still present, becomes less rapid than a power law as previously noticed in previous RG investigations ${ }^{26 \mid 55]}$ This results from the weakening of the two-loop singularity and modification of Cooper and Peierls channels interference by coherent warping of the FS. However, as discussed in more details below, when temperature is further decreased toward the instabilities lines, but still in the normal phase, the quasi- particle weight decay develops quite distinctive features as a function of the antinesting parameter $t_{\perp}^{\prime}$. The influence of the latter becomes in its turn non perturbative, especially in the normal phase of the superconducting domain of the phase diagram.

Below $T_{X}$, the position of minima in $z\left(k_{\perp}\right)$ gradually shifts to $k_{\perp}= \pm \pi / 4$ and $k_{\perp}= \pm 3 \pi / 4$, as the details of the Fermi surface become coherent. The shift can be understood from the nesting condition of the whole spectrum, which reads

$$
E_{+}\left(\mathbf{k}+\mathbf{q}_{0}\right)=-E_{-}(\mathbf{k})+\delta\left(k_{\perp}\right) .
$$

Here $\delta\left(k_{\perp}\right)=4 t_{\perp}^{\prime} \cos 2 k_{\perp}$ is the $k_{\perp}$-dependent deviation from perfect nesting. The minima coincide with the loci, $k_{\perp}= \pm \pi / 4$, and $\pm 3 \pi / 4$ on the FS where $\delta( \pm \pi / 4)$ and $\delta( \pm 3 \pi / 4)$ vanish and perfect nesting conditions prevail. Conversely, at $k_{\perp}= \pm \pi / 2,0$, and $\pm \pi, \delta\left(k_{\perp}\right)= \pm 4 t_{\perp}^{\prime}$, and nesting deviations, like $z\left(k_{\perp}\right)$, are maximum below $T_{X}$. As the temperature comes close to $T_{\mathrm{SDW}}, z\left(k_{\perp}\right)$ enters in the critical SDW regime and falls off toward zero due to singular scattering amplitudes which signal the breakdown of the perturbative RG procedure (Figs. 6 and 7).

Considering now the SC domain of the phase diagram at $t_{\perp}^{\prime}>t_{\perp}^{\prime *}$, the variation of $z\left(k_{\perp}\right)$ with respect to both $k_{\perp}$ and $T$ is similar to the one encountered in the SDW case at high $T \gg t_{\perp}^{\prime}$. As $T$ decreases well below $t_{\perp}^{\prime}$, however, $z\left(k_{\perp}\right)$ behaves differently.

Let us first examine the $k_{\perp}$ dependence. From Fig. 6 $\mathrm{b}$, the modulation of $z\left(k_{\perp}\right)$ in the normal state is roughly similar to that obtained in the SDW case with a slight modulation in $k_{\perp}$ that persists down to the $\mathrm{CW}$ regime (Fig. 6). In this temperature domain and down to $T_{c}$, the system is governed by the SCd fixed point and the maxima at $k_{\perp}= \pm \pi / 2$ remain stationary at the approach of $T_{c}$, consistently with the location of nodes for the SCd gap below $T_{c}$. The amplitude of the peaks at $k_{\perp}=0$ and $\pm \pi$ present a steady reduction as the temperature is lowered.

Turning to the $T$ dependence in the SCd sector of the phase digram. We see from Fig. 7 that not too far from $t_{\perp}^{\prime *}$ at the intermediate $t_{\perp}^{\prime}=26.8 \mathrm{~K}, z\left(k_{\perp}\right)$ exhibits a significative decay in the metallic state extending far above the critical domain, comprising the entire $\mathrm{CW}$ regime of spin correlations. The amplitude of the $z$ decay correlates with the one of SDW fluctuations in the normal phase as $t_{\perp}^{\prime}$ is tuned away from $t_{\perp}^{\prime *}$. When $t_{\perp}^{\prime}$ is far upward, at $35 \mathrm{~K}$, for instance, one has $T_{c}=0.12 \mathrm{~K} \ll T_{c}^{*}$, and a very weak temperature dependence is found for $z$ above $T_{c}$, which is very close to that of a Fermi liquid (Fig. 7).

If we now concentrate on the $\mathrm{CW}$ temperature interval, $T_{c} \lesssim T \leq T_{\mathrm{CW}}$, we see that the $z$ decay at $k_{\perp}=0$ for instance, can be seen as a result of logarithmic corrections that conform to the expression,

$$
z\left(k_{\perp}\right)=\frac{z_{0}\left(k_{\perp}\right)}{1+g\left(k_{\perp}\right) \ln \left(T_{\mathrm{CW}} / T\right)},
$$




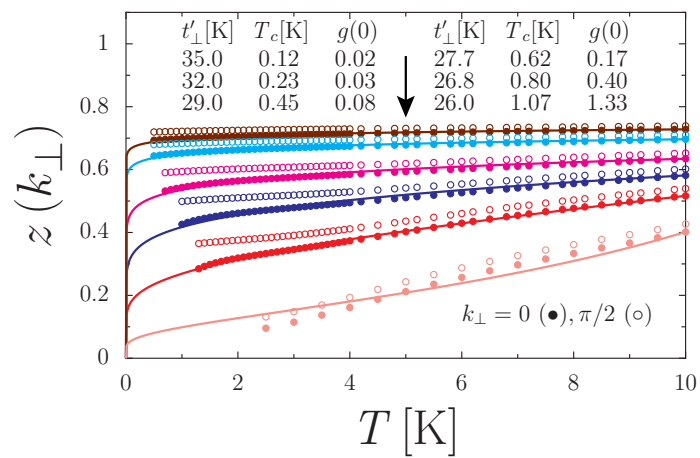

FIG. 8: (Color on line) Low temperature dependence quasiparticle weight $z\left(k_{\perp}\right)$ for different antinesting parameters $t_{\perp}^{\prime}$ for $k_{\perp}=0$ (full circles), and nodal point $\pi / 2$ (open circles). The continuous lines correspond to a fit to Eq. 118 of the marginal liquid theory at $k_{\perp}=0$.

which is compatible with the phenomenology proposed by the marginal Fermi liquid theory for the quasiparticle self-energy in two dimensions. ${ }^{[56]}$ Here $z_{0}\left(k_{\perp}\right)=$ $z\left(k_{\perp}, T_{\mathrm{CW}}\right)$ is the quasi-particle weight at the cutoff temperature $T_{\mathrm{CW}}=10 \mathrm{~K}$, and $g\left(k_{\perp}\right)$ is the square of an effective (averaged over two momentums variables) coupling constant normalized by $\pi v_{F}$.

For most values of $t_{\perp}^{\prime}$ shown in Fig. 8, the RG results in the $\mathrm{CW}$ regime for $z$ at $k_{\perp}=0$ are compatible with an effective marginal Fermi liquid behavior, characterized by a weak coupling value for $g\left(k_{\perp}=0\right)$. As we will see in more details below (Sec. IV C 3), such a behavior is in line with the presence of a $T$-linear component in the temperature dependent electron-electron scattering rate for the same values of $t_{\perp}^{\prime}$; a similar connection between $z$ and the scattering rate is well known in the context of marginal Fermi liquid theory ${ }^{[56}$ According to Fig. 8 , by raising $t_{\perp}^{\prime}$ the reduction of $z$ declines, but the negative curvature is apparently maintained. In the limit of large $t_{\perp}^{\prime}$ or small $T_{c}, z$ becomes ultimately constant in temperature with $g(0) \rightarrow 0$, as expected for a Fermi liquid.

From Fig. 8, however, the quality of the fit wanes significantly near the critical value $t_{\perp}^{\prime *}$. At $t_{\perp}^{\prime}=26 \mathrm{~K}$ for example, the reduction of $z$ becomes rather important at low temperature with a $g(0)$ value exceeding unity, as a result of large positive feedback of Cooper pairing on SDW fluctuations that lead the scattering amplitudes to flow to very strong coupling. This signals that using one-loop RG calculations in the one-particle two-loop self-energy may become insufficient. We will see indeed in Sec. IV C 3 that the pronounced reduction in $z$ coincides with a marked rising of the scattering rate showing a progressive suppression of quasi-particles akin to a pseudogap behavior. ${ }^{[57} \mathrm{A}$ full two-loop RG calculation of both self-energy and scattering amplitudes is required to overcome this difficulty at $t_{\perp}^{\prime} \approx t_{\perp}^{\prime *} \cdot \underline{47}$

Not too close to $t_{\perp}^{*}$, the marginal Fermi liquid fit works equally well for all $k_{\perp}$, with the exception of the nodal points, $\pm \pi / 2$, of the superconducting SCd gap below $T_{c}$.

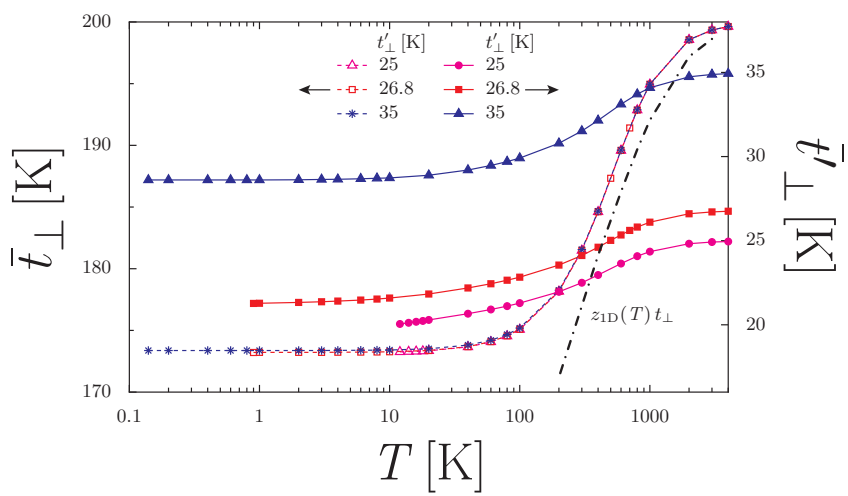

FIG. 9: (Color on line) Renormalized interchain hopping $\bar{t}_{\perp}$ and antinesting term $\bar{t}_{\perp}^{\prime}$ by self-energy corrections as a function of temperature and for different bare values of $t_{\perp}^{\prime}$. The dashed dot line corresponds to the result of extended scaling hypothesis for the renormalization of $t_{\perp}$.

As shown in Fig. 8 there is a small positive curvature that develops for $z(\pi / 2)$ as $T$ approaches $T_{c}$. A Fermi liquid component is forming at those nodal points where $z( \pm \pi / 2) \rightarrow$ constant (Fig. 8 and Figs. 766 b).

\section{B. Renormalization of transverse hopping integrals}

The corrections produced by the flow of self-energy generate the new term, $z_{\perp}\left(\bar{k}_{\perp}\right)$, in the single particle propagator 14) (see also App. A). In the static limit, $z_{\perp}\left(k_{\perp}\right) \equiv z_{\perp}\left(k_{\perp}, \omega_{\nu=0}\right)$ affects the transverse part of the electron spectrum and then deforms the Fermi surface. According to (14), the modification of the transverse energy is of the form, $\delta \varepsilon_{\perp}\left(k_{\perp}\right)=z\left(k_{\perp}\right) z_{\perp}\left(k_{\perp}\right)$, which as an even function of $k_{\perp}$ can be decomposed into the Fourier series

$$
\begin{aligned}
\delta \varepsilon_{\perp}\left(k_{\perp}\right)=t_{0} & +\delta t_{\perp} \cos k_{\perp}+\delta t_{\perp}^{\prime} \cos 2 k_{\perp} \\
& +\delta t_{\perp}^{\prime \prime} \cos 3 k_{\perp}+\ldots .
\end{aligned}
$$

The series coefficients consist of corrections to the chemical potential $\left(t_{0}\right)$, the first $\left(\delta t_{\perp}\right)$, second $\left(\delta t_{\perp}^{\prime}\right)$, third $\left(\delta t_{\perp}^{\prime \prime}\right) \ldots$, nearest-neighbors hopping integrals of the transverse direction. Here $\delta t_{\perp}^{\prime \prime}$, as well as higher order harmonics are new terms of the spectrum generated by the RG flow. It turns out, however, that in all cases, the total correction $\delta \varepsilon_{\perp}$ is very accurately fitted to the existing form of the transverse part of the spectrum (4), which involves $\delta t_{\perp}$ and $\delta t_{\perp}^{\prime}$ alone; the values of $t_{0}, \delta t_{\perp}^{\prime \prime}$, etc .., are vanishingly small and can thus be disregarded as irrelevant terms.

Adding both corrections to the spectrum leads to the temperature dependent renormalisation of interchain hoppings, which become $\bar{t}_{\perp}=t_{\perp}+\delta t_{\perp}$ and $\bar{t}_{\perp}^{\prime}=t_{\perp}^{\prime}+\delta t_{\perp}^{\prime}$. The temperature dependences of $\bar{t}_{\perp}$ and $\bar{t}_{\perp}^{\prime}$ are shown in Fig. 9 for different bare $t_{\perp}^{\prime}$ values. According to the Figure, one observes a downward shift of $\bar{t}_{\perp}$, taking place above $T_{X}$ where it follows a decay of the 
form $\bar{t}_{\perp} \sim z_{1 D}(T) t_{\perp}$. In the 1D temperature domain the amplitude of $\bar{t}_{\perp}$ is therefore governed by the reduction of the quasi-particle weight (dashed dotted line of Fig. 9p, as predicted long ago by the extended scaling hypothesis $24 \mid 27$. The downward renormalization, which is about $13 \%$ of the bare $t_{\perp}$ for the model parameters used, is essentially $t_{\perp}^{\prime}$ independent and does not correlate with $T_{c}$. The renormalization of $t_{\perp}$ is taking place for the most part above $T_{X}$ and is a function of the initial couplings. The present approach can describe continuously the whole temperature domain including the crossover toward the coherent transverse single particle motion below $T_{X}$ where the renormalization of $\bar{t}_{\perp}$ levels off (Fig. 9); this occurs despite a quasi-particle weight that still decreases below $T_{X}$ (Fig. 7).

If we now turn to the reduction of the antinesting term $t_{\perp}^{\prime}$, it is small in the $1 \mathrm{D}$ temperature regime and becomes significant below $T_{X}$ where the details of the Fermi surface become coherent. The amplitude of the reduction attained is about $15-20 \%$ of the bare value at very low temperature and all $t_{\perp}^{\prime}$.

\section{Retarded one-particle self-energy}

From the expression (14) of the Green function, the $\ell \rightarrow \infty$ Matsubara self-energy at arbitrary $\omega_{\nu}$ on the FS is given by

$$
\Sigma_{p}\left(\bar{k}_{\perp}\right)=i \omega_{\nu}\left[1-z\left(\bar{k}_{\perp}\right)\right]-z_{\perp}\left(\bar{k}_{\perp}\right)
$$

Using a Padé approximants procedure, $\Sigma_{p}\left(k_{\perp}, i \omega_{\nu}\right)$ is analytically continued to the retarded form $\Sigma_{p}^{\mathrm{R}}\left(k_{\perp}, \omega\right)=$ $\Sigma_{p}^{\prime}\left(k_{\perp}, \omega\right)+i \Sigma_{p}^{\prime \prime}\left(k_{\perp}, \omega\right)$ on the real $\omega$ axis, made up of the real $\left(\Sigma_{p}^{\prime}\right)$ and imaginary $\left(\Sigma_{p}^{\prime \prime}\right)$ parts. The analytical continuation is carried out for a discrete set of $N_{\omega}$ Matsubara frequencies, whose distribution is peaked around $\nu=0$ and spread with a decreasing density as $\nu$ grows to large values. To obtain $\Sigma_{p}^{\mathrm{R}}\left(k_{\perp}, \omega\right)$ over the whole frequency range, extending up to $\pm \frac{3}{2} E_{0}(=9000 \mathrm{~K})$ with respect to the bare Fermi level at $\omega=0$, good convergence in the $\omega$ dependence is achieved for $N_{\omega} \sim 20$ or so. However, when focussing on the behavior of spectral quantities for small energy intervals centered on $\omega=0$, the most accurate results are obtained at smaller $N_{\omega} \sim 10$ close to $\nu=0$.

\section{Real and Imaginary parts}

Typical results for $\Sigma_{+}^{\prime}\left(k_{\perp}=0, \omega\right)$ and $\Sigma_{+}^{\prime \prime}\left(k_{\perp}=0, \omega\right)$ over the whole frequency range are shown in Fig. 10 in the SDW and SCd sectors of the phase diagram. The general features shown correspond to those expected for a metal with single particle excitations. The real part $\Sigma_{+}^{\prime}$ for example, presents two minima and maxima, and crosses the Fermi level linearly with a negative slope, as

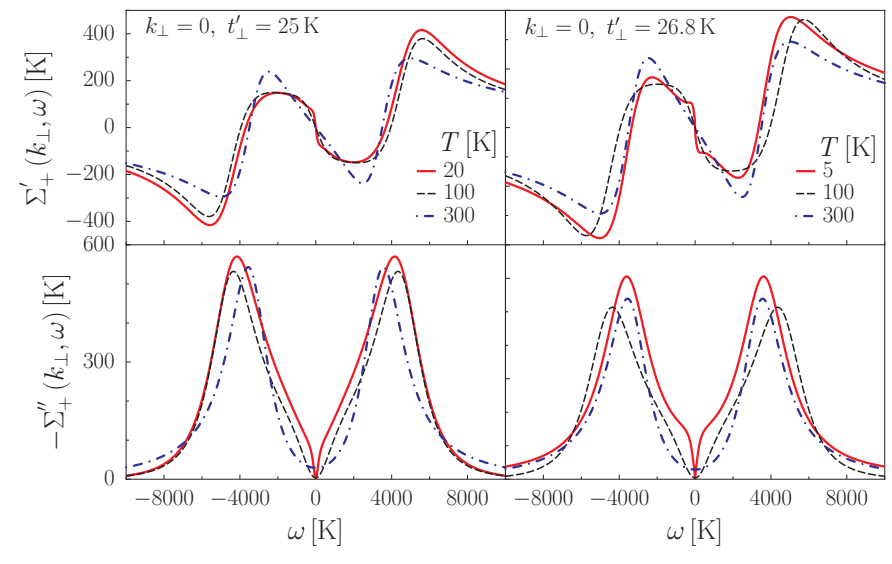

FIG. 10: (Color on line) Typical frequency dependence of the real $\left(\Sigma^{\prime}\right)$ and imaginary $\left(\Sigma^{\prime \prime}\right)$ parts of the one-particle selfenergy at different temperatures in the $\operatorname{SDW}\left(t_{\perp}^{\prime}=25 \mathrm{~K} ; T=\right.$ $20,100,300 \mathrm{~K}$, left $)$ and $\mathrm{SC}\left(t_{\perp}^{\prime}=26.8 \mathrm{~K} ; T=5,100,300 \mathrm{~K}\right.$, right) parts of the phase diagram in Fig. 4

$\omega \rightarrow 0$. To the resonant structure of $\Sigma_{+}^{\prime}$ at low frequency corresponds a minimum in minus the imaginary part, $-\Sigma_{+}^{\prime \prime}$ at $\omega=0$, as shown in Fig. 10 .

The slope in $\Sigma_{+}^{\prime}$ at $\omega \rightarrow 0$ enters in the determination of the quasi-particle weight by the Fermi liquid expression

$$
z\left(k_{\perp}\right)=\left[1-\left(\partial \Sigma_{+}^{\prime}\left(k_{\perp}, \omega\right) / \partial \omega\right)_{\omega \rightarrow 0}\right]^{-1} .
$$

Thus the accuracy of the analytical continuation can be verified by comparing $(21)$ to the result discussed previously following the direct evaluation of Eq. [15). In all cases studied, both determinations match to a very good degree of accuracy (one percent or less of discrepancy at arbitrary $T$ ).

In the $1 \mathrm{D}$ temperature domain at $T=300 \mathrm{~K}>T_{X}$ and for both values of $t_{\perp}^{\prime}$, the linear part of $\Sigma_{+}^{\prime}$ that crosses the origin spreads over the large energy interval, $\delta \omega \sim 2 \pi T$ (Fig. 10). In this temperature range, the quasi-particle weight essentially coincides with its $1 D$ limit, $z_{1 \mathrm{D}}$, which varies as a power law in temperature consistently with the results of Sec. IV A (Fig. 7). To the $\omega$-linear behavior within $\delta \omega$ in $\Sigma_{+}^{\prime}$ corresponds a rounded minimum centered at $\omega=0$ for $-\Sigma_{+}^{\prime \prime}$, well described by the quadratic dependence, $-\Sigma_{+}^{\prime \prime} \propto \omega^{2}-$ known to hold for a Fermi liquid 59 . So despite the Luttinger liquid power law reduction of $z_{1 D}$ in temperature, Fermi liquid type corrections in frequency remain within a finite energy interval fixed by the temperature. Similar Fermi liquid corrections in frequency around the FS can be found in the exact spectral quantities of the 1D Tomanaga-Luttinger model at finite $T$.6061

As the temperature is lowered below $T_{X}$, the width $\delta \omega$ where the linear part of $\Sigma_{+}^{\prime}$ takes place narrows markedly. The slope at $\omega \rightarrow 0$ becomes steeper, which in accordance to (21) is indicative of a decreasing quasi-particle weight, as obtained from (15) and Fig. 7. The narrow width of the $\omega$-linear variation in $\Sigma_{+}^{\prime}$ is still correlated with 

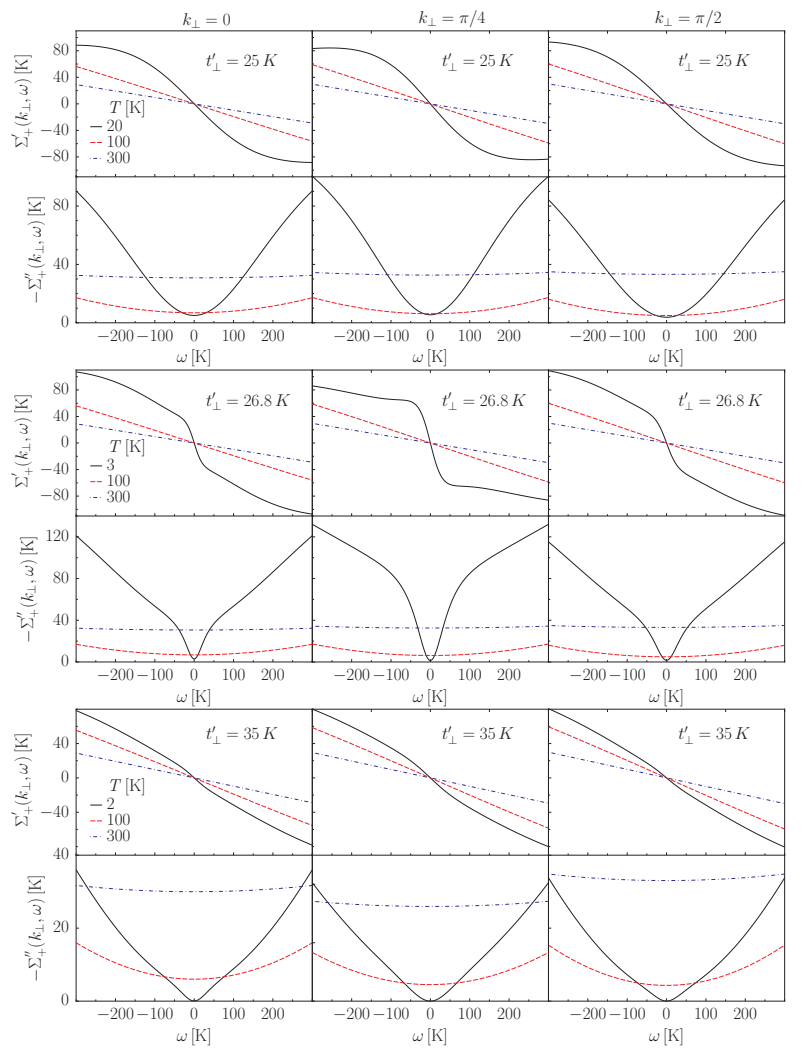

FIG. 11: (Color line) Low frequency dependence of the real $\left(\Sigma_{+}^{\prime}\right)$ and imaginary $\left(\Sigma_{+}^{\prime \prime}\right)$ parts of the one-particle self-energy at different $t_{\perp}^{\prime}$ and regions of the Fermi surface.

the quadratic frequency dependence in $-\Sigma_{+}^{\prime \prime}$ (Fig. 10). Outside $\delta \omega$, however, the dip structure in $-\Sigma_{+}^{\prime \prime}$ sharpens considerably and is no longer quadratic in frequency.

The modification of the quasi-particle resonance at low temperature can be examined in more details by focussing on low frequency dependence of $\Sigma_{+}^{\prime}$ and $-\Sigma_{+}^{\prime \prime}$, in the range $|\omega| \lesssim \pi T_{X}$ and for different $t_{\perp}^{\prime}$, as shown in Fig. 11. According to the Figure, we verify that for all $t_{\perp}^{\prime}$, the quasi-particle resonance shape in $\Sigma_{+}^{\prime}$ modifies and crosses linearly the origin with a slope that becomes steeper as $T$ is lowered within $\delta \omega$. The slope along with the amplitude of the resonance in $\Sigma_{+}^{\prime}$ are the most pronounced close to $t_{\perp}^{\prime *}$ and fall off as $t_{\perp}^{\prime}$ moves far apart from the SDW-SCd juncture, that is when $T_{c}$ becomes small.

Well below $T_{X}$, the modification of $\omega$-linear term in $\Sigma_{+}^{\prime}$ co-occurs with the development of a narrow dip in $-\Sigma_{+}^{\prime \prime}$ that invariably shows a quadratic dependence within $\delta \omega$. The expression for the real part,

$$
\Sigma_{+}^{\prime}\left(k_{\perp}, \omega\right) \approx-\lambda\left(k_{\perp}, T\right) \omega, \quad(|\omega|<\delta \omega / 2),
$$

though linear in $\omega$ at small frequency, as in a Fermi liquid, has a momentum dependent linear coefficient $\lambda\left(k_{\perp}, T\right)$ related to the anomalous decay of quasi-particle weight $\left(\lambda=z^{-1}-1\right)$. As we have seen previously, $\lambda\left(k_{\perp}, T\right)=$ $g\left(k_{\perp}\right) \ln T_{\mathrm{CW}} / T$ in the $\mathrm{CW}$ regime of the SCd sector and

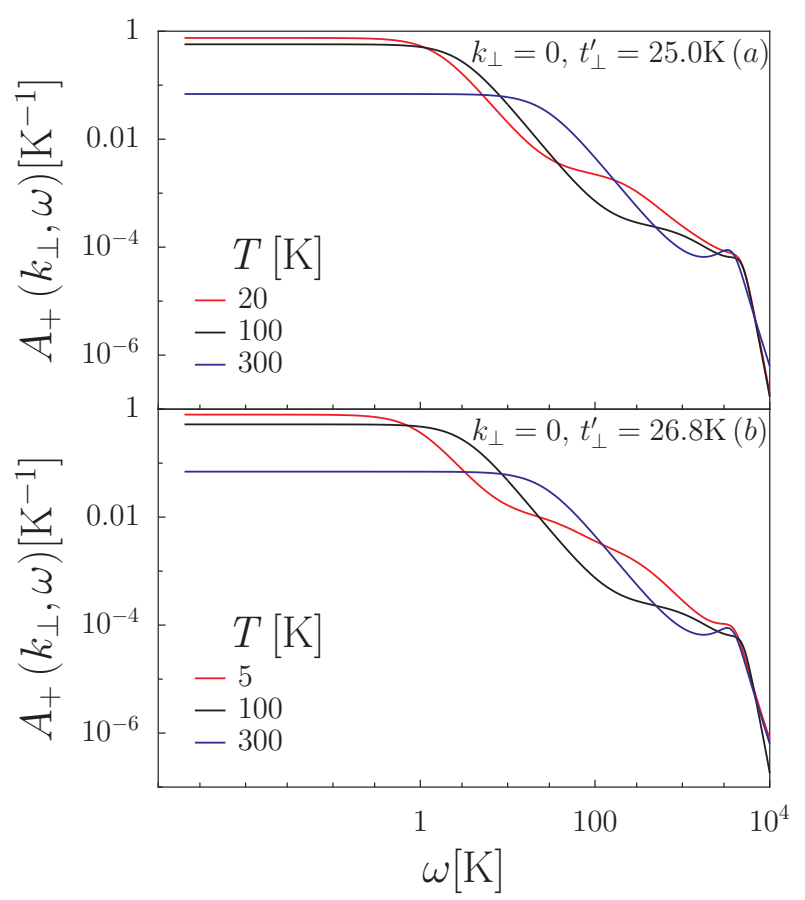

FIG. 12: (Color on line) Spectral weight at $k_{\perp}=0$ on the Fermi surface versus frequency (log-log scale) in the metallic domain of the SDW $\left(t_{\perp}^{\prime}=25 \mathrm{~K}\right)$ and SCd $\left(t_{\perp}^{\prime}=26.8 \mathrm{~K}\right)$ sectors of the phase diagram.

is well approximated by marginal Fermi liquid logarithmic corrections (Fig. 8).

Well outside the range of frequency fixed by $\delta \omega$ at low temperature, deviations to linearity in $\Sigma_{+}^{\prime}$ yield as much departures from quadratic variations in $-\Sigma_{+}^{\prime \prime}$ (Fig. 11). The latter are rather well described by a linear frequency dependence of the form, $-\Sigma_{+}^{\prime \prime}\left(k_{\perp}, \omega\right) \approx$ $\alpha_{0}\left(k_{\perp}\right)+\alpha\left(k_{\perp}\right) \mid \omega \underline{62}$. The extrapolation shows a finite intercept as $\omega \rightarrow 0\left(\alpha_{0} \neq 0\right)$, due to the $\omega^{2}$ dependence within $\delta \omega$. The size of the linear factor $\alpha\left(k_{\perp}\right)$ falls off as $t_{\perp}^{\prime}$ is increasing or $T_{c}$ decreasing.

Regarding the $k_{\perp}$ dependence exhibited by $\Sigma_{+}^{\prime}$ and $\Sigma_{+}^{\prime \prime}$, it is present albeit not large. The steepest slope at zero frequency is found at $k_{\perp}=\pi / 4$ (and similarly at $-\pi / 4, \pm 3 \pi / 4)$, in agreement with the minima of $z\left(k_{\perp}\right)$ found in Fig. 6 and that are most pronounced close to $t_{\perp}^{\prime *}$.

\section{Spectral weight}

The nature of single particle excitations on the FS as a function of the antinesting parameter $t_{\perp}^{\prime}$ or $T_{c}$ can be further sharpened by looking at the spectral weight $A_{p}\left(\mathbf{k}_{F, p}, \omega\right)=-2 \operatorname{Im} G_{p}^{\mathrm{R}}\left(\mathbf{k}_{F, p}, \omega\right)$, defined with the aid of the retarded Green function. For $p=+$, for instance, one has

$$
A_{+}\left(k_{\perp}, \omega\right)=\frac{-2 \Sigma_{+}^{\prime \prime}\left(k_{\perp}, \omega\right)}{\left[\omega-\Sigma_{+}^{\prime}\left(k_{\perp}, \omega\right)\right]^{2}+\left[\Sigma_{+}^{\prime \prime}\left(k_{\perp}, \omega\right)\right]^{2}} .
$$




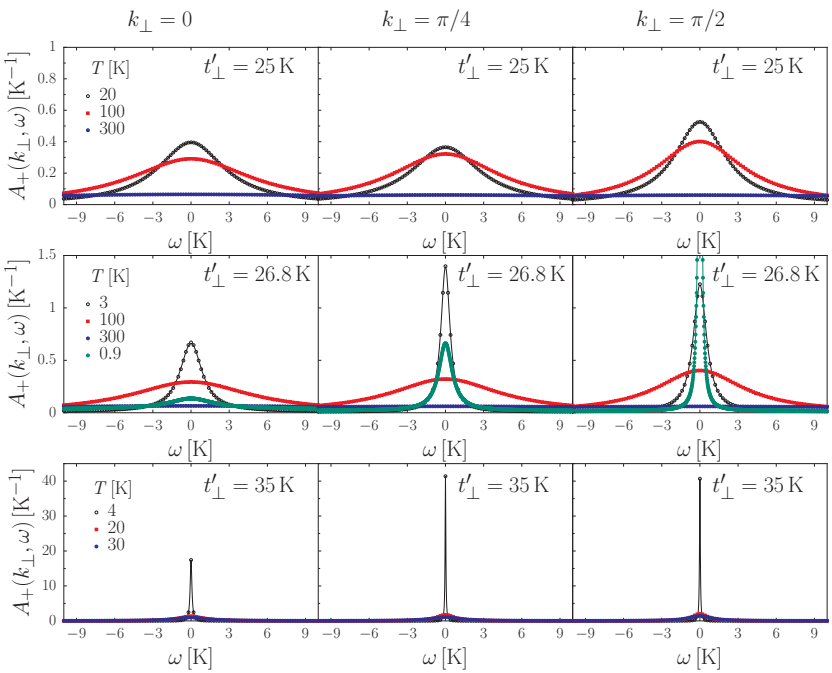

FIG. 13: (Color on line) Low frequency dependence of the single particle spectral weight at different $t_{\perp}^{\prime}$ and regions of the Fermi surface.

Typical normal state profiles of $A_{+}\left(k_{\perp}=0, \omega\right)$ over the whole range of frequencies are shown in Fig. 13 at different $T$ on each side of $t_{\perp}^{\prime *}$.

For both $t_{\perp}^{\prime}$, one finds a growing peak at $\omega=0$ as $T$ decreases while its width in frequency is reduced. From Fig. 12, one also notices that with decreasing $T$ the spectral weight is redistributed over a sizable part of the frequency range. Although the redistribution is not large in amplitude, the shift of spectral density toward high frequencies reflects the influence of interactions in the build-up of an incoherent part of the spectrum in the mid-frequency range, which is concomitant with the lost of quasi-particle weight $z\left(k_{\perp}\right)$ on the FS associated to the coherent counterpart (Fig. 7). The redistribution is consistent with the sum rule,

$$
(2 \pi)^{-1} \int_{-\infty}^{+\infty} A_{+}\left(k_{\perp}, \omega\right) d \omega=1,
$$

which is accurately satisfied as a function of $k_{\perp}$, for arbitrary $T$ and $t_{\perp}^{\prime}$.

In Fig. 13, we present a close-up view of the coherent component of the spectral weight at small frequency for different $t_{\perp}^{\prime}, T$, and $k_{\perp}$. We see that on the SDW side at low temperature (top panel of Fig. 13), the height of the central peak in $A_{+}\left(k_{\perp}, \omega\right)$ is maximum at $k_{\perp}=\pi / 2$, whereas the minimum is at $k_{\perp}=\pi / 4$. With roughly the same width $\Delta \omega$ in frequency, these peaks correlate with the minimum (maximum) found in the quasi-particle weight for the best (worst) nesting conditions (Fig. 6), through the Fermi liquid relation

$$
z\left(k_{\perp}\right)=(2 \pi)^{-1} \int_{\Delta \omega} A_{+}\left(k_{\perp}, \omega\right) d \omega .
$$

However, as we enter the low temperature metallic phase of the SCd sector of the phase diagram at intermediate and large $t_{\perp}^{\prime}$, there is a notable change in the positions of maxima and minima of $A_{+}\left(k_{\perp}, \omega\right)$ along the Fermi surface. Under the control of the SCd fixed point in the $\mathrm{CW}$ domain, the quasi-particle peak becomes according to Fig. 13 maximum at $k_{\perp}=\pi / 4$ (also at $-\pi / 4, \pm 3 \pi / 4$ ) and minimum at $k_{\perp}=0$ (also at $\pm \pi$ ). The width of the peaks and the tails, though remaining sizable at very low temperature, progressively narrow while the height raise as $t_{\perp}^{\prime}$ moves away from $t_{\perp}^{\prime *}$. It is only in the limit of large $t_{\perp}^{\prime}$ or very small $T_{c}$ that the peaks evolve toward the formation of a Dirac like structure at $\omega=0$ (Fig. 13 , bottom panel). Put together with the very weak temperature decay of the quasi-particle weight (Figs. 7 and 8 ) and the dominant quadratic temperature dependence of the scattering rate (see Sec. IV C 3), this limit is symptomatic of the emergence of a Fermi liquid. A similar Fermi liquid recovery for quasi-particles on the SCd side is found at the nodal points, $k_{\perp} \pm \pi / 2$, as $T \rightarrow T_{c}$, while for the rest antinodal region, the spectral weight is suppressed by critical fluctuations preceding the opening of the d-wave gap (Fig. 13 middle).

\section{Electron-electron scattering rate}

An important quantity giving key information on the impact of correlations on single particle properties on the FS is the electron-electron scattering rate. This quantity is defined by the imaginary part of the self-energy at $\omega=0$,

$$
\tau_{k_{\perp}}^{-1}=-2 \Sigma_{+}^{\prime \prime}\left(k_{\perp}, 0\right)
$$

In Fig. 14 the $\tau_{k_{\perp}}^{-1}$ temperature dependence at $k_{\perp}=0$ is shown for $t_{\perp}^{\prime}$ in the SDW $\left(t_{\perp}^{\prime}=25 \mathrm{~K}\right)$ and $\mathrm{SCd}\left(t_{\perp}^{\prime}=\right.$ $26.8-35 \mathrm{~K})$ regions of the phase diagram.

For SDW, $\tau_{k_{\perp}=0}^{-1}$ decreases from high temperature and ultimately shows an upturn at low temperature at the approach of the critical domain of $T_{\mathrm{SDW}}(\simeq 12 \mathrm{~K})$, where couplings become strong and short-range SDW fluctuations act as a singular source of scattering for quasiparticles. On the SCd side, $\tau_{k_{\perp}=0}^{-1}$ slowly decreases with decreasing temperature, then crosses over toward the $\mathrm{CW}$ regime below 10K (Fig. 4 , where $\tau_{k_{\perp}=0}^{-1}$ falls off toward zero. The drop corresponds to the sharpening of the coherent peak in the spectral weight for the same temperature interval (Fig. 13); it continues down to the onset of critical SCd domain close to $T_{c}$ where a sudden rise in $\tau_{k_{\perp}=0}^{-1}$ occurs (not shown in the Figure).

Within the $\mathrm{CW}$ temperature domain, the scattering rate is well described by a polynomial temperature dependence of the form

$$
\tau_{k_{\perp}}^{-1} \approx a\left(k_{\perp}\right) T+b\left(k_{\perp}\right) T^{2},
$$

which comprises an anomalous linear component and quadratic Fermi liquid term. The fit of the $k_{\perp}=0$ results to the above expression in the normal phase interval $\left.] T_{c}, 4 \mathrm{~K}\right]$ of the $\mathrm{CW}$ domain reveals a pronounced 

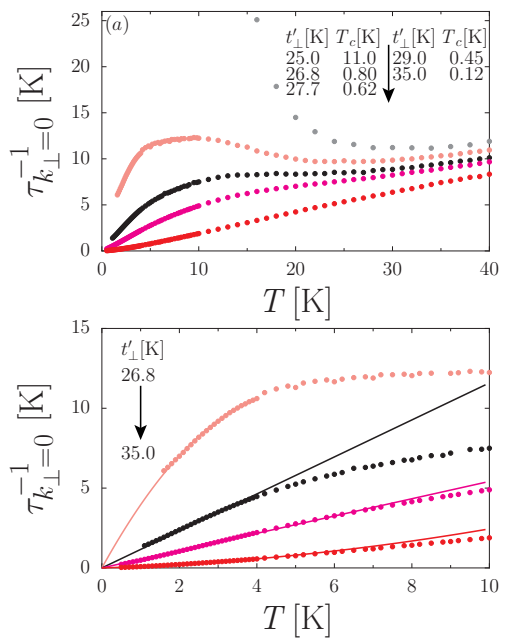

FIG. 14: (Color on line) (a) : Temperature dependence of the electron-electron scattering rate at $k_{\perp}=0$ for different $t_{\perp}^{\prime}$ in the SDW and SCd regions of the phase diagram; (b) : Curie-Weiss temperature region in the SCd domain where the continuous lines correspond to a fit to Eq. 25).

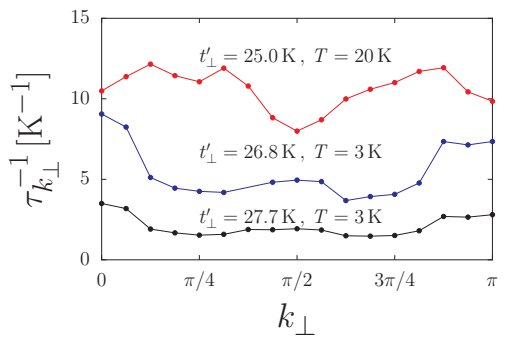

FIG. 15: (Color on line) Momentum dependence of the quasiparticle scattering rate on the Fermi surface in the SDW ( $\left.t_{\perp}^{\prime}=25 \mathrm{~K}\right)$ and $\mathrm{SCd}\left(t_{\perp}^{\prime}=26.8\right.$ and $\left.27.7 \mathrm{~K}\right)$.

linear component with the $a\left(k_{\perp}=0\right)$ coefficient that decreases with $t_{\perp}^{\prime}$ or the decay of $T_{c}$ (Figs. 14. b and 17. a). The Fermi liquid component is only emerging well above $t_{\perp}^{\prime *}$ where $\tau_{k_{\perp}=0}^{-1}$ begins to show a positive curvature. In the limit of large $t_{\perp}^{\prime}$ or small $T_{c}$, the anomalous part is vanishingly small and only the Fermi liquid contribution remains, in concordance with the virtually temperature independent quasi-particle weight (Figs. 7.8) and the delta-like peak in the spectral weight (Fig. 13).

It is instructive to look at the $k_{\perp}$ dependence of the scattering rate for both sectors of the phase diagram. As shown in Fig. 15 for the SDW metallic phase, there is a correspondence between the best (worst) nested regions of the FS at $k_{\perp} \simeq \pm \pi / 4$ or $\pm 3 \pi / 4( \pm \pi / 2)$ where $\tau_{k_{\perp}}^{-1}$ is maximum (minimum) and those where $z\left(k_{\perp}\right)$ is minimum (maximum) (Fig. 6). This is consistent with the usual picture of 'warmer' regions of the FS governed by stronger scattering on SDW fluctuations and better nesting conditions. By contrast, this correspondence no longer holds in the CW metallic regime on the SCd side: the best nested regions are where the scattering rate is
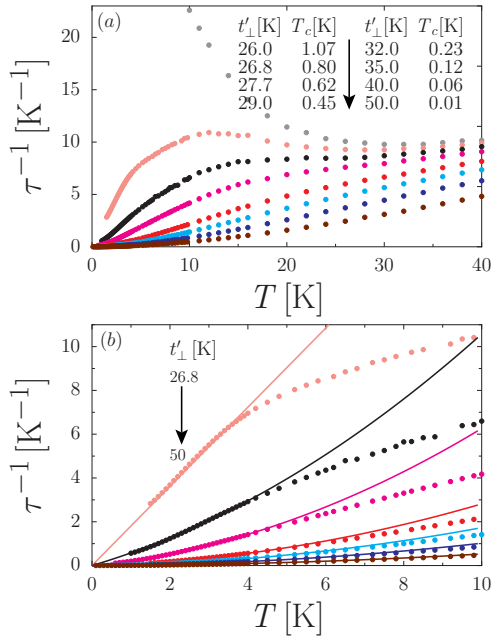

FIG. 16: (Color on line) (a) : Temperature dependence of the electron-electron scattering rate averaged on the Fermi surface for different $t_{\perp}^{\prime}$ in the superconducting sector of the phase diagram; (b): polynomial fit (continuous lines) of the temperature dependence of the mean scattering rate (Eq. 26).

the lowest: the maxima being shifted at $k_{\perp}=0$ and $\pm \pi$ (Fig. 15). Thus, although SDW correlations remain the strongest in the metallic state, that is $\chi_{\mathrm{SDW}} \gg \chi_{\mathrm{SCd}}$ at all $T$ (except very close to $T_{c}$ ), the SDW source of scattering is clearly affected by the presence of Cooper pairing. This shift in the $\tau_{k_{\perp}}^{-1}$ maxima is consistent with the one encountered for the spectral weight as $t_{\perp}^{\prime}$ crosses over to the SCd side (Fig. 13).

In spite of the $k_{\perp}$ anisotropy shown by $\tau_{k_{\perp}}^{-1}$ in Fig. 15 . the temperature dependence along the Fermi surface turns out to be similar as a function of $k_{\perp}$, only the amplitude of the $a\left(k_{\perp}\right)$ and $b\left(k_{\perp}\right)$ of the polynomial form (25) varies. It is therefore useful to concentrate on the averaged scattering rate, $\tau^{-1} \equiv\left\langle\tau_{k_{\perp}}^{-1}\right\rangle_{\mathrm{FS}}$, over the FS. The average scattering rate is shown in Fig. 16 at different $t_{\perp}^{\prime}>t_{\perp}^{\prime *}$ in the superconducting domain.

Consider first the SCd region close to the juncture with SDW, near the critical $t_{\perp}^{\prime *}$. As shown in Fig. 16. the scattering rate decreases from high temperature, but ultimately shows an upturn with lowering $T$, which is typical of a SDW type of behavior (see Fig. 14 a). In spite of an SCd instability toward superconductivity at $T_{c} \sim 1 \mathrm{~K}$, SDW correlations are so large that the scattering becomes critical or 'insulating' like. This strong coupling behavior accords with the marked suppression of quasi-particle weight discussed for the same $t_{\perp}^{\prime}$ conditions in Fig. 8. Strong coupling effects close to $t_{\perp}^{\prime *}$ may indicate a pseudogap type behavior and signal the limitations of weak coupling one-loop calculations for scattering amplitudes.

As $t_{\perp}^{\prime}$ is gradually tuned away from $t_{\perp}^{\prime *}$, the $\tau^{-1}$ variation modifies around 10K. Following the example of the $k_{\perp}=0$ case in Fig. 14, as $T \rightarrow 0, \tau^{-1}$ extrapolates to- 


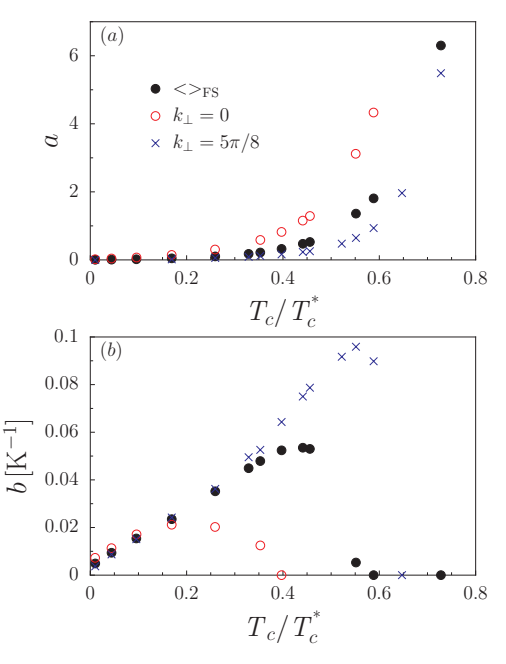

FIG. 17: (Color on line) Evolution of the linear (a) and quadratic (b) coefficients of the scattering rate as a function of the ratio $T_{c} / T_{c}^{*}$ for $k_{\perp}=0$ (open circles) and $k_{\perp}=5 \pi / 8$ (crosses). The full circles correspond to the averages $a$ and $b$ over the Fermi surface (Eq. 26.

ward zero in the $\mathrm{CW}$ domain. Metallic one-particle states then sharpen with a steadily growing lifetime down to the onset of critical SCd fluctuations (Fig. 16). The corresponding temperature dependence of $\tau^{-1}$ is rather well described by the averaged polynomial form of $25{ }^{63}$,

$$
\tau^{-1} \approx a T+b T^{2}
$$

where $a=\left\langle a\left(k_{\perp}\right)\right\rangle_{\mathrm{FS}}$ and $b=\left\langle b\left(k_{\perp}\right)\right\rangle_{\mathrm{FS}}$ are the coefficients averaged over the FS. Therefore both a Fermi liquid and an anomalous linear term superimpose in $\tau^{-1}$, as shown by the continuous lines of Fig. 16 b obtained from a fit of $(26)$ to the $\tau^{-1}$ results in the same CW temperature interval, $\left.] T_{c}, 4 \mathrm{~K}\right]$, used previously for $k_{\perp}=0$.

Not too far from the boundary, there is a finite $t_{\perp}^{\prime}$ region where the quadratic part vanishes and $\tau^{-1}$ is uniquely $T$-linear. By averaging the scattering rate, this region is much than for $k_{\perp}=0$ (Fig. 17-b).

A few remarks are in order concerning the origin of a $T$-linear component in $\tau^{-1}$. It can be seen as the result of quasi-particles scattering on SDW correlations whose spectral density peaked at frequency lower or of the order of temperature, which would be compatible with the sizable amplitude of $\tilde{\chi}_{\text {SDW }}$ or large SDW correlation length $\xi$ (Fig. 4). In these conditions, electron scattering on spin fluctuations in two dimensions ${ }^{64 \mid 65}$, is known to yield $\tau^{-1} \sim T$ when $\xi$ is weakly temperature dependent, which is here realized for $T \ll \Theta$. An alternative but related interpretation of the impact of vertex parts on self-energy diagrams of Fig. 2 is provided by the phenomenology of the marginal Fermi liquid. In the latter scheme, electron scattering on dispersionless bosons formed by particlehole pair excitations also yields $T$-linear scattering rate $[56$ Such an interpretation would be compatible with the temperature decay of the quasi-particle weight, which, as we have seen previously, follows the marginal Fermi liquid behavior in the same temperature domain of the normal phase (Fig. 8).

As $t_{\perp}^{\prime}$ is tuned upward and $T_{c}$ decreases, there is a threshold in $t_{\perp}^{\prime}$ above which the variation of $\tau^{-1}$ begins to develop a finite curvature well described by a Fermi liquid $b T^{2}$ term (Fig. 16). According to Fig. 17, the quadratic coefficient is first growing rapidly from zero, reaches a maximum and ultimately decreases as one moves away from the SDW-SCd juncture and $T_{c}$ drops along the $t_{\perp}^{\prime}$ axis; this non monotonic trace is likely to result from the interplay between the stiffening of the spin fluctuations spectrum (increasing $\Theta$, Fig. 4a) and their decreasing amplitude.

As shown in Fig. 17, the emergence of a Fermi liquid coefficient $b$ co-occurs with the weakening of the linear component as $t_{\perp}^{\prime}$ increases or $T_{c}$ decreases. Remarkably, however, the $T$-linear term persists over a large interval of $t_{\perp}^{\prime}$ where $T_{c}$ takes appreciable values (Fig. 17). This 'correlation' between $a$ and $T_{c}$ demarcates from what is usually expected from a quantum critical point driven by the SDW channel of fluctuations alone. In the latter situation, the influence of superconducting pairing is absent and the linear component of the scattering rate is ordinarily confined within a cone of quantum criticality close to the critical value of the tuning parameter 16 though recent calculations show some departure from this scheme within the framework of the single SDW fluctuation channel 57 . The presence of Cooper-Peierls pairing mixing is here responsible for the expanse of the $T$-linear component along the $t_{\perp}^{\prime}$ axis. The possibility in the RG framework to switch off all Cooper pairing contributions in the scattering amplitudes (8) allows a direct confirmation of this mutual influence. When all Cooper loops in (8) are put to zero and the Peierls channel is singled out, we verify that linearity is indeed confined to a narrow region above $t_{\perp}^{\prime *}$, and is further followed by the prompt emergence of a Fermi liquid $b T^{2}$ term, with a $b$ coefficient an order of magnitude larger than the one found when the two scattering channels are present (Fig. 17).

Another revealing fingerprint of the contribution of Cooper pairing to the broad $t_{\perp}^{\prime}$-range of anomalous scattering rate can be found in the anisotropy of the linear$T$ coefficient, $a\left(k_{\perp}\right)$, along the FS. As shown in Fig. 18 for different $t_{\perp}^{\prime}$, a prominent maximum with secondary maxima are found at $k_{\perp}=0$ and $k_{\perp}= \pm \pi$ respectively. These positions are clearly where the superconducting $\mathrm{d}$ wave gap, $\Delta\left(k_{\perp}\right)=\Delta_{0} \cos k_{\perp}+\mathcal{O}\left(\cos 2 k_{\perp}\right)$, is expected to be maximum in amplitude below $T_{c}$. The minima of $a\left(k_{\perp}\right)$ are found relatively close to the nodal region for the gap at $k_{\perp} \simeq \pm 5 \pi / 8$ whose variation with $T_{c}$ is also shown in Fig. 17.

This contrasts with the situation prevailing at either high temperature, namely above the $\mathrm{CW}$ temperature domain, or in the metallic state of the SDW sector of the phase diagram where $\tau_{k_{\perp}}^{-1}$ is peaked in the neighborhood of the best nesting points at $k_{\perp}= \pm \pi / 4$ or $\pm 3 \pi / 4$ (Fig. 15). 


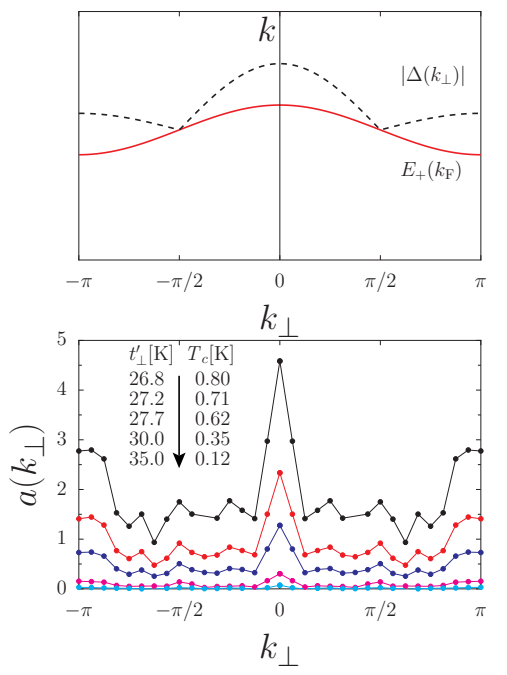

FIG. 18: (Color on line) Not to scale variation of the dwave superconducting gap modulus, $\left|\Delta\left(k_{\perp}\right)\right|$, along the $+k_{F}$ Fermi surface sheet (top); $T$-linear coefficient anisotropy of the quasi-particle scattering rate along the Fermi surface and at different $t_{\perp}^{\prime}$ (bottom).

\section{DISCUSSION AND CONCLUDING REMARKS}

In the normal phase associated to the SDW-SCd sequence of instabilities of the quasi-1D electron gas, the above RG analysis brought out a number of anomalies in the single particle quantities. For weakly dimerized chains responsible for small Umklapp scattering, and relatively weak electron-electron couplings, the temperature scales for instabilities are occurring far below the single particle crossover scale $T_{X} \sim t_{\perp}$, which expands the normal phase at lower temperature where single particle anomalies become under the growing influence of nesting deviations $t_{\perp}^{\prime}$.

By tuning nesting deviations along the $t_{\perp}^{\prime}$ axis, the critical downturn of $T_{\mathrm{SDW}}$ merges to the superconducting instability line at a much smaller $T_{c}$. The normal phase becomes therefore enlarged by the Curie-Weiss interval of staggered spin fluctuations. As shown by the temperature dependent SDW susceptibility, it extends up to $T_{\mathrm{CW}} \sim 10 \mathrm{~K}$, a scale equal to many times $T_{c}$. This temperature region turns out to be distinct in that $T_{\mathrm{CW}}$ corresponds to the scale where the SDW fixed point becomes unstable and another fixed point is emerging in the SCd channel. The evolution of the flow in the CW domain is rather gradual, however, as shown by the continuous transformation of interactions in momentum space. Besides Umklapp, the RG flow shows that normal scattering amplitudes at the SDW momentum transfer, $\mathbf{q}_{0}=\left(2 k_{F}, \pi\right)$, are reinforced in the antinodal region of the FS, while the nodal counterpart sees the scattering weakened ${ }^{[19}$ Such an interplay is the fingerprint of a steady constructive interference between Cooper (SCd) and Peierls (SDW) pairings that takes place in the CW temperature interval. It correlates in amplitude with the distance from the critical $t_{\perp}^{\prime *}$ for the occurrence of superconductivity. When fed into the two-loop one-particle self-energy, it yields the scaling of number of NFL quasiparticles properties with $T_{c}$.

The fact that two simultaneous pairing processes interfere with and distort each other makes difficult a standard description of low energy excitations of the normal phase in terms of long wavelength order parameter fluctuations. ${ }^{14115}$ In this regard, it is of interest to compare the $\mathrm{CW}$ situation with the one that prevails in 1D systems where such entanglement of many-body processes is at its maximum. These optimal conditions turn out to be realized here when $T \gg T_{X}$, which are well known to invalidate the Ginzburg-Landau-Wilson description of collective excitations in terms of order parameter fluctuations. The proper collective excitations in the $1 \mathrm{D}$ range are essentially given by the bosonic modes of the Luttinger liquid, which is quantum critical in character at all scales 54 In the low temperature regime, at $T \ll T_{X}$, the coherent warping of the FS and nesting deviations do attenuate interference, which becomes non uniform on the FS. As stressed above, in spite of this distortion, its presence is maintained so that the mixing of electron-hole and electron-electron excitations carries over the flaws of the usual Ginzburg-Landau-Wilson description of correlations at lower temperature. When factored in, interference then produces an expanded range of quantum criticality for the normal state and introduces an effective correlation of quasi-particle anomalies with $T_{c}$.

Extended quantum criticality is particularly well illustrated by the NFL downturn of the quasi-particle weight $z\left(k_{\perp}\right)$ in the $\mathrm{CW}$ temperature domain. We have seen that for the whole non nodal part of the FS, $z\left(k_{\perp}\right)$ is well described by temperature dependent logarithmic corrections similar to those found in the marginal Fermi liquid theory. Since the phenomenology of the marginal Fermi liquid is typically quantum critical in character $\frac{56 \mid 66}{, 6}$ the persistent NFL properties shown over a broad range of $t_{\perp}^{\prime}$ or $T_{c}$ is compatible with an expanded region of quantum criticality in the phase diagram of the quasi-1D electron gas.

Another related feature shared with this phenomenology is supplied by the existence of a linear- $T$ component in the polynomial temperature dependence of the scattering rate $\tau_{k_{\perp}}^{-1}$. Following the example of quasi-particle weight fraction, the strength of linear- $T$ scattering within the same CW region of the normal phase also scales with $T_{c}$ along the $t_{\perp}^{\prime}$ axis.

These findings on scattering rate primarily apply to the problem of anomalous electric transport in the metallic state of (TMTSF $)_{2} X$ salts. Within the Curie-Weiss regime of SDW fluctuations determined by $\mathrm{NMR}^{\sqrt[46 \mid 8]{618}}$ the resistivity data of prototype compounds like $X=\mathrm{PF}_{6}$ and $\mathrm{ClO}_{4}$ 11112|67, were shown to be well described by the polynomial form, $\Delta \rho \approx A T+B T^{2}$ (after subtraction of the elastic contribution from impurities). From the 
critical pressure where superconductivity joins to antiferromagnetism in the phase diagram, $\frac{1168}{168}$ the linear coefficient $A$ shows a clear scaling with $T_{c}$. Under pressure, it becomes superimposed to a Fermi liquid contribution that is quadratic in $T$. The latter ultimately dominates the temperature dependence of resistivity in the high pressure limit where the materials are no longer superconducting.

The above features of electrical resistivity in the Bechgaard salts adhere in many respects to the behavior shown by the scattering rate as a function of $t_{\perp}^{\prime}$ in the present model. In the presence of Umklapp scattering, the inelastic contribution to resistivity in the absence of vertex corrections can be written as $\Delta \rho=$ $4 \pi\left\langle\tau_{k_{\perp}}\right\rangle_{\mathrm{FS}}^{-1} / \omega_{p}^{2},\left(\omega_{p}\right.$ is the plasma frequency), which is related to the inverse scattering time averaged over the FS - the latter differing very little from $\left\langle\tau_{k_{\perp}}^{-1}\right\rangle_{\mathrm{FS}}$ considered in Sec. IV C 35|12. Under these conditions, it follows that the problem of anomalous linear- $T$ resistivity and its correlation to $T_{c}$ for (TMTSF) $)_{2} X$ can be viewed as the consequence of an expanded quantum critical regime for electrons that scatter off antiferromagnetic fluctuations. The underlying interference of pairings may reveal to be a key determinant in the mechanism that connects scattering to $T_{c}$ in the Bechgaard salts.

This brings us to consider whether similar ideas can go beyond the sole confines of low- $T_{c}$ materials and apply to other unconventional superconductors. In this respect iron-based pnictide superconductors are of particular interest. There are indeed striking similarities shown by the SDW-SC sequence of ground states in many pnictides, provided that $T_{c}$ and $T_{\mathrm{SDW}}$ of the Bechgaard salts are magnified by a factor 20 or so. The scaling of both linear- $T$ resistivity, ${ }^{10|11| 13}$ and Curie-Weiss regime of spin fluctuations with $T_{c}$, over a wide range of atomic substitution in pnictides, ${ }^{69 \mid 70}$ suggest that the mechanism described here for extended criticality may be of more general importance for the normal state of superconductors near a SDW instability. Despite a difference in their FS (more 2D, multiple sheets), the two basic active ingredients for extended criticality, namely, nesting and pairing, are known to be both present and interfering $171+73$.

The role of Cooper pairing in anomalous quantum criticality may be also relevant to high- $T_{c}$ cuprates. In holetype materials, for instance, common features with the organics can be found in the overdoped region, namely where where the FS is large and coherent and the pseudo gap is absent. The hole-doped materials are among the first compounds showing systematic scaling of anomalous resistivity with $T_{c}, \frac{10 / 1374 / 75}{2}$ a temperature scale that can be two orders of magnitude larger than for the Bechgaard salts. Nevertheless, as a function of hole concentration, resistivity evolves from perfectly $T$-linear, to the purely quadratic dependence of the Fermi liquid once superconductivity is suppressed in the strongly overdoped region. ${ }^{74 / 77}$ Results of functional $R G$ on the $2 \mathrm{D}$ Hubbard model indicate that spin fluctuations which mix with electron-electron scattering processes of large mo- mentum transfer can yield a linear component in the scattering rate whose amplitude correlates with band filling and therefore $T_{c}{ }^{78}$ In the framework of the two-particle self-consistent approach to the antiferromagnetic channel of 2D Hubbard model, $\frac{64}{6}$ the calculation of resistivity, which takes into account vertex corrections and the extended range of spin fluctuation effects in $2 \mathrm{D}$, shows the presence of anomalous temperature dependence for resistivity over a sizable domain of carrier concentration. 57

For electron-doped cuprates, $79 \sqrt{81}$ antiferromagnetism borders on superconductivity as a function of electron concentration, in a way analogous to the Bechgaard salts under pressure. In these cuprates too, definite $T$-linear resistivity is observed close to optimal doping $\sqrt{82}$; it gradually weakens upon further doping to ultimately disappear with the emergence of Fermi liquid scattering when superconductivity is no longer found at high enough carrier concentration. ${ }^{[83}$ The well defined Fermi surface at optimal $n$-doping with hot spots of antiferromagnetic scattering suggests that mutual reinforcement with dwave Cooper pairing should also come into play in these materials. ${ }^{84}$ Furthermore from a high order RGcalculations, the interplay between cold and hot regions of a two-dimensional Fermi surface is shown to be favorable to unconventional criticality and NFL behavior over extended regions of the $\mathrm{FS} .8586$

The connection between anomalous resistivity and Cooper pairing in cuprates has been further emphasized recently from the anisotropy displayed by the scattering rate, as extracted from angular dependent magnetoresistance ${ }^{87 \mid 88}$ Anisotropy of the scattering rate in the copper oxides planes was found to simulate the angular profile of the SCd gap amplitude. A RG analysis of the 2D Hubbard model close to half-filling has shown that some mutual influence of electron-hole and electron-electron scattering channels at large momentum transfer can reproduce the d-wave like modulation of the scattering rate. 7884

In this respect the parallel with the results obtained here for the $\tau_{k_{\perp}}^{-1}$ anisotropy in the quasi-1D case deserves to be underlined. In Fig. 18 the amplitude of the linear- $T$ scattering was found to mimic the variation of the d-wave gap modulus on the open FS. The anisotropy emerges in the CW domain of regular spin fluctuations, namely where pairing interference modifies the momentum dependence of interactions. This shows up in the scattering rate on the $\mathrm{FS}$, which sees the cooling of nodal regions concomitant with the warming of regions where the $\mathrm{d}$ wave gap is maximum.

Before closing this discussion, it is worth coming back to the limitations of the present approach when antinesting is tuned sufficiently close to the critical $t_{\perp}^{*}$ for superconductivity and the scaled scattering amplitudes become sufficiently large that they invalidate the quasiparticle picture on the Fermi surface. Although these strong coupling effects disclose the limitations of one-loop approximation for the couplings entering in two-loop selfenergy calculations, they may suggest, however, that the 
conditions for a pseudogap formation are combined. Actually at the approach of $t_{\perp}^{\prime *}$, the sharp drop of $T_{\mathrm{SDW}}$ indicates that the latter should be linked to a narrow $t_{\perp}^{\prime}$-interval from $t_{\perp}^{\prime *}$ where SDW fluctuations effects are large. Within this interval, the whole Curie-Weiss temperature domain would be characterized by near critical SDW fluctuations likely to destroy quasi-particle excitations. Such a weak coupling RG signature of a pseudogap regime is comparable to the one discussed in the context of the $2 \mathrm{D}$ Hubbard model near half-filling. ${ }^{89190}$ A possible route to a more controlled description of this region of the phase diagram would be to push the RG technique beyond the one-loop level for both self-energy and scattering amplitudes at finite temperature.26|44|52|91] 93 Such an extension is under current investigation. $\frac{47}{6}$

In conclusion, we have used the weak coupling RG method to investigate the normal phase of quasi-1D superconductors near a spin-density-wave instability. Interference between electron-electron and electron-hole pairings, which enters in the mechanism of superconductivity and enhancement of spin fluctuations, has been found to play a major role in the NFL character of the normal state. The tuning of nesting deviations was shown to modify interference between the two scattering channels and as a result the strength of anomalies in single particle quantities. A correlation of the superconducting instability line with non Fermi liquid behavior was then found to develop. It can be seen as an extended quantum critical region of the phase diagram that goes beyond the usual confines of an ending magnetic critical point. This mechanism of extended criticality may be a key factor in the evolution of linear- $T$ resistivity not only in the Bechgaard salts under pressure, but also in other unconventional superconductors such as pnictides and cuprates.

\section{Acknowledgments}

C. B thanks S. Brown, N. Doiron-Leyraud, Y. Fuseya, D. Jerome, Y. Suzumura, L. Taillefer, M. Tsuchiizu and A.-M. Tremblay for comments and discussion on several aspects of this work. C. B also expresses its gratitude to the the National Science and Engineering Research Council of Canada (NSERC), the Réseau Québcois des Matériaux de Pointe (RQMP) and the Quantum materials program of Canadian Institute of Advanced Research (CIFAR) for financial support. Computational resources were provided by the Réseau québécois de calcul de haute performance (RQCHP) and Compute Canada.

\section{Appendix A: One-particle self- energy}

In this appendix, we detail the calculation of the finite temperature outer shell contribution to the one-particle self-energy in the Kadanoff-Wilson scheme of RG. Four our purposes, it is convenient to discretize the steps of mode elimination by the partial trace operation 44152 .

$$
\begin{aligned}
& \delta \Sigma_{+}^{(1)}\left(\bar{k}_{\perp}\right)= \\
& \sum_{n=1}^{N} \sum_{m=n}^{N}(\stackrel{-}{3} \\
& \delta \Sigma_{+}^{(2)}\left(\bar{k}_{\perp}\right)=
\end{aligned}
$$

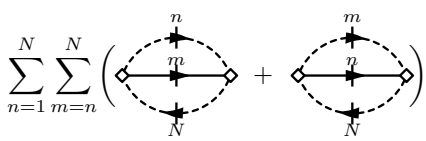

$$
\begin{aligned}
& \delta \Sigma_{+}^{(3)}\left(\bar{k}_{\perp}\right)=
\end{aligned}
$$

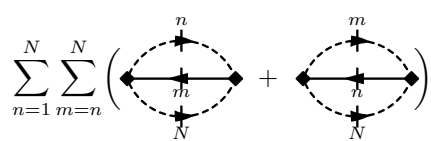

FIG. 19: Outer shell contribution to the one-particle selfenergy of the $p=+$ branch for normal $\left(\delta \Sigma_{+}^{(1,2)}\right)$ and Umklapp $\left(\delta \Sigma_{+}^{(3)}\right)$ scattering processes. $n$ and $m$ refer to the energy indexes in the cascades of contraction, and $N$ to the index of the running energy shell at $E_{N}=E_{0}(\ell)$.

Thus after $N \gg 1$ steps of mode elimination, the scaled energy bandwidth will be noted $E_{N}\left(\equiv E_{0}(\ell)\right)$. Each step consists of mode integration within an infinitesimal shell $E_{\Delta}$ in the $E$-space, namely

$$
E_{\Delta} \equiv \frac{E_{0}-E_{N}}{N}
$$

where $E_{0}=2 E_{F}$; here an equidistant step is chosen for simplicity. After $n$ steps the energy distance, $E_{n}$, from the Fermi surface is

$$
E_{n}=\frac{E_{0}-n E_{\Delta}}{2}, \quad 1 \leq n \leq N .
$$

We define the $n$-th energy shell interval $\mathcal{O}_{n}$

$$
\mathcal{O}_{n} \equiv\left[-E_{n-1},-E_{n}\right] \cup\left[E_{n}, E_{n-1}\right] \equiv \mathcal{O}_{n}^{-} \cup \mathcal{O}_{n}^{+}
$$

as the union of shells below and above the Fermi level. After the cascade of contractions on the three-particles interactions generated by the RG flow, one obtains the one-particle self-energy corrections of Fig. 19. These consist of three types of one-particle self-energy corrections, $\delta \Sigma_{+}=\sum_{i=1}^{3} \delta \Sigma_{+}^{(i)}$, whose diagrammatic representation of is shown in Fig. 19. Here $\delta \Sigma_{+}^{(1,2)}$ are associated to the forward and backward scattering contributions and $\delta \Sigma_{+}^{(3)}$ to Umklapp scattering. Their explicit expressions are given by 


$$
\begin{aligned}
\delta \Sigma_{+}^{(1)}\left(\bar{k}_{\perp}\right)= & \frac{1}{2}\left(\frac{\pi v_{F} T}{L}\right)^{2} \iint \frac{d k_{\perp}^{\prime}}{2 \pi} \frac{d q_{\perp}}{2 \pi} \sum_{\{k, \omega\}} \sum_{n, m}\left(-g_{2}^{2}\left(\widetilde{k}_{\perp 1}\right)-g_{1}^{2}\left(\widetilde{k}_{\perp 1}\right)+g_{2}\left(\widetilde{k}_{\perp 1}\right) g_{1}\left(\widetilde{k}_{\perp 1}\right)\right) \\
& \times G_{-}^{0}\left(\bar{k}^{\prime}\right) G_{-}^{0}\left(\bar{k}^{\prime}+\bar{q}\right) G_{+}^{0}(\bar{k}+\bar{q}) \vartheta_{N}\left(E_{-}\left(\mathbf{k}^{\prime}\right)\right)\left(\vartheta_{m}\left(E_{+}(\mathbf{k}+\mathbf{q})\right) \vartheta_{n}\left(E_{-}\left(\mathbf{k}^{\prime}+\mathbf{q}\right)\right)+\left\{\vartheta_{n} \leftrightarrow \vartheta_{m}\right\}\right), \\
\delta \Sigma_{+}^{(2)}\left(\bar{k}_{\perp}\right)= & \frac{1}{2}\left(\frac{\pi v_{F} T}{L}\right)^{2} \iint \frac{d k_{\perp}^{\prime}}{2 \pi} \frac{d q_{\perp}}{2 \pi} \sum_{\{k, \omega\}} \sum_{n, m}\left(-g_{2}^{2}\left(\widetilde{k}_{\perp 2}\right)-g_{1}^{2}\left(\widetilde{k}_{\perp 2}\right)+g_{2}\left(\widetilde{k}_{\perp 2}\right) g_{1}\left(\widetilde{k}_{\perp 2}\right)\right) \\
& \times G_{-}^{0}\left(\bar{k}^{\prime}\right) G_{-}^{0}\left(\bar{k}^{\prime}+\bar{q}\right) G_{+}^{0}(\bar{k}-\bar{q}) \vartheta_{N}\left(E_{-}\left(\mathbf{k}^{\prime}\right)\right)\left(\vartheta_{m}\left(E_{+}(\mathbf{k}-\mathbf{q})\right) \vartheta_{n}\left(E_{-}\left(\mathbf{k}^{\prime}+\mathbf{q}\right)\right)+\left\{\vartheta_{n} \leftrightarrow \vartheta_{m}\right\}\right), \\
\delta \Sigma_{+}^{(3)}\left(\bar{k}_{\perp}\right)= & \frac{1}{2}\left(\frac{\pi v_{F} T}{L}\right)^{2} \iint \frac{d k_{\perp}^{\prime}}{2 \pi} \frac{d q_{\perp}}{2 \pi} \sum_{\{k, \omega\}} \sum_{n, m}\left(-g_{3}^{2}\left(\widetilde{k}_{\perp 3}\right)-g_{3}^{2}\left(\widetilde{k}_{\perp 4}\right)+g_{3}\left(\widetilde{k}_{\perp 3}\right) g_{3}\left(\widetilde{k}_{\perp 4}\right)\right) \\
& \times G_{-}^{0}\left(\bar{k}^{\prime}\right) G_{-}^{0}\left(-\bar{k}^{\prime}+\bar{q}\right) G_{+}^{0}(-\bar{k}+\bar{q}) \vartheta_{N}\left(E_{-}\left(\mathbf{k}^{\prime}\right)\right)\left(\vartheta_{m}\left(E_{+}(-\mathbf{k}+\mathbf{q})\right) \vartheta_{n}\left(E_{-}\left(-\mathbf{k}^{\prime}+\mathbf{q}\right)\right)+\left\{\vartheta_{n} \leftrightarrow \vartheta_{m}\right\}\right)
\end{aligned}
$$

where $\vartheta_{n}(E)=1$ for $E \in \mathcal{O}_{n}$, and 0 otherwise; $\widetilde{\vartheta}_{n}(E)=$ $\sum_{m=n}^{N} \vartheta_{m}(E)=1$ for $E_{N}<|E| \leq E_{n}$, and 0 otherwise. The wave vectors are defined as

$$
\begin{aligned}
\widetilde{k}_{\perp 1} & =\left(k_{\perp}+q_{\perp}, k_{\perp}^{\prime}, k_{\perp}\right), \\
\widetilde{k}_{\perp 2} & =\left(k_{\perp}-q_{\perp}, k_{\perp}^{\prime}+q_{\perp}, k_{\perp}\right), \\
\widetilde{k}_{\perp 3} & =\left(k_{\perp},-k_{\perp}+q_{\perp}, k_{\perp}^{\prime}\right), \\
\widetilde{k}_{\perp 4} & =\left(k_{\perp},-k_{\perp}+q_{\perp},-k_{\perp}^{\prime}+q_{\perp}\right),
\end{aligned}
$$

Note that the use of the propagator 14 in the calculation of the diagrams of Fig. 19 introduces products of the form $g_{i} \cdot z \cdot z$. In one-loop scheme for interactions, these $z$ factors in the self-energy correction are evaluated in the non interacting limit, that is $z=1$. Likewise, the calculation of self-energy is done with respect to the bare Fermi surface by using the bare propagator in the calculations for which all the $z_{\perp}$ in $(14)$ are fixed to zero.

Following the discretization of the $k_{\perp}$ interval into $N_{p}$ $(=32)$ patches, such as $k_{\perp}^{\prime}, q_{\perp}=-\frac{N_{p}}{2} \Delta_{\perp}, . .,\left(\frac{N_{p}}{2}-1\right) \Delta_{\perp}$, $\Delta_{\perp}=\frac{2 \pi}{N p}$, the expression becomes

$$
\begin{aligned}
\delta \Sigma_{+}^{(1)}\left(\bar{k}_{\perp}\right)= & \frac{1}{2} \iint \frac{d k_{\perp}^{\prime}}{2 \pi} \frac{d q_{\perp}}{2 \pi} \\
& \times\left(-g_{2}^{2}\left(\widetilde{k}_{\perp 1}\right)-g_{1}^{2}\left(\widetilde{k}_{\perp 1}\right)+g_{2}\left(\widetilde{k}_{\perp 1}\right) g_{1}\left(\widetilde{k}_{\perp 1}\right)\right) \\
& \times\left(\mathcal{I}_{1}^{1}\left(\widetilde{k}_{\perp}, i \omega_{\nu}\right)+\mathcal{I}_{1}^{2}\left(\widetilde{k}_{\perp}, i \omega_{\nu}\right)\right) .
\end{aligned}
$$

The expression for $\mathcal{I}_{1}^{1}$ is given by

$$
\begin{aligned}
& \mathcal{I}_{1}^{1}\left(\tilde{k}_{\perp}, i \omega_{\nu}\right)=\left(\frac{\pi v_{F} T}{L}\right)^{2} \\
& \times \int_{k_{\perp}^{\prime}}^{k_{\perp}^{\prime}+\Delta_{\perp}} \int_{q_{\perp}}^{q_{\perp}+\Delta_{\perp}} \frac{d k_{\perp}^{\prime \prime}}{2 \pi} \frac{d q_{\perp}^{\prime}}{2 \pi} \\
& \times \sum_{\{k, \omega\}} \sum_{n, m} G_{-}^{0}\left(\bar{k}^{\prime \prime}\right) G_{-}^{0}\left(\bar{k}^{\prime \prime}+\bar{q}^{\prime}\right) G_{+}^{0}\left(\bar{k}+\bar{q}^{\prime}\right) \\
& \times \vartheta_{N}\left(E_{-}\left(\mathbf{k}^{\prime \prime}\right)\right) \vartheta_{m}\left(E_{-}\left(\mathbf{k}^{\prime \prime}+\mathbf{q}^{\prime}\right)\right) \vartheta_{n}\left(E_{+}\left(\mathbf{k}+\mathbf{q}^{\prime}\right)\right)
\end{aligned}
$$

which obeys $\mathcal{I}_{1}^{2}=\left.\mathcal{I}_{1}^{1}\right|_{\vartheta_{n} \leftrightarrow \vartheta_{m}}$, and where

$$
\widetilde{k}_{\perp}=\left(k_{\perp}, k_{\perp}^{\prime}, q_{\perp}\right)
$$

Carrying out the frequency summations, we obtain:

$$
\begin{aligned}
& T^{2} \sum_{\{\omega\}} G_{-}^{0}\left(\bar{k}^{\prime \prime}\right) G_{-}^{0}\left(\bar{k}^{\prime \prime}+\bar{q}^{\prime}\right) G_{+}^{0}\left(\bar{k}+\bar{q}^{\prime}\right) \\
& =\left[n_{B}\left(E_{-}\left(\mathbf{k}^{\prime \prime}+\mathbf{q}^{\prime}\right)-E_{-}\left(\mathbf{k}^{\prime \prime}\right)\right)+n_{F}\left(E_{+}\left(\mathbf{k}+\mathbf{q}^{\prime}\right)\right)\right] \\
& \times \frac{\left[n_{F}\left(E_{-}\left(\mathbf{k}^{\prime \prime}+\mathbf{q}^{\prime}\right)\right)-n_{F}\left(E_{-}\left(\mathbf{k}^{\prime \prime}\right)\right)\right]}{i \omega_{\nu}+E_{-}\left(\mathbf{k}^{\prime \prime}+\mathbf{q}^{\prime}\right)-E_{+}\left(\mathbf{k}^{\prime \prime}\right)-E_{+}\left(\mathbf{k}+\mathbf{q}^{\prime}\right)}
\end{aligned}
$$

where $n_{F}(E)\left(n_{B}(E)\right)$ is the Fermi (Bose) distribution factor. For a given function of energy $f(E)$, we have for the outer-shell integral:

$$
\frac{\pi v_{F}}{L} \sum_{k^{\prime \prime}} \vartheta_{N}\left(E_{-}\left(\mathbf{k}^{\prime \prime}\right)\right) f\left(E_{-}\left(\mathbf{k}^{\prime \prime}\right)\right)=\frac{\delta E_{0}(\ell)}{2} \sum_{\mu= \pm} f\left(\mu E_{N}\right)
$$


which yields

$$
\begin{aligned}
\delta \Sigma_{+}^{(1)}\left(\bar{k}_{\perp}\right) & =\frac{1}{2} \iint \frac{d k_{\perp}^{\prime}}{2 \pi} \frac{d q_{\perp}}{2 \pi} \\
\times & \times\left(-g_{2}^{2}\left(\widetilde{k}_{\perp 1}\right)-g_{1}^{2}\left(\widetilde{k}_{\perp 1}\right)+g_{2}\left(\widetilde{k}_{\perp 1}\right) g_{1}\left(\widetilde{k}_{\perp 1}\right)\right) \\
\times & \left(-\left[G_{+}^{0}(\bar{k})\right]^{-1} I_{1}\left(\widetilde{k}_{\perp}, i \omega_{\nu}\right) d \ell+I_{1}^{\prime}\left(\widetilde{k}_{\perp}, i \omega_{\nu}\right) d \ell\right)
\end{aligned}
$$

The expressions for $I_{1}$ and $I_{1}^{\prime}$ are

$$
\begin{aligned}
I_{1}\left(\widetilde{k}_{\perp}, i \omega_{\nu}\right) & =\frac{E_{0}(\ell)}{4} \sum_{\mu= \pm} \iint \frac{d k_{\perp}^{\prime \prime}}{2 \pi} \frac{d q_{\perp}^{\prime}}{2 \pi} \int_{E_{N}+\left|q_{\mu 1}\right| / 2}^{E_{0}-\left|q_{\mu 1}\right| / 2} d q^{\prime} \\
& {\left[\mathcal{B}\left(k_{\perp}^{\prime \prime}, q_{\perp}^{\prime}, q^{\prime}-q_{\mu 1} / 2\right)+\mathcal{B}\left(k_{\perp}^{\prime \prime}, q_{\perp}^{\prime},-q^{\prime}-q_{\mu 1} / 2\right)\right] }
\end{aligned}
$$

and

$$
\begin{aligned}
I_{1}^{\prime}\left(\widetilde{k}_{\perp}, i \omega_{\nu}\right)= & \frac{E_{0}(\ell)}{4} \sum_{\mu= \pm} \iint \frac{d k_{\perp}^{\prime \prime}}{2 \pi} \frac{d q_{\perp}^{\prime}}{2 \pi} \int_{E_{N}+\left|q_{\mu 1}\right| / 2}^{E_{0}-\left|q_{\mu 1}\right| / 2} d q^{\prime} \\
& {\left[\mathcal{A}\left(k_{\perp}^{\prime \prime}, q_{\perp}^{\prime}, q^{\prime}-q_{\mu 1} / 2\right)+\mathcal{A}\left(k_{\perp}^{\prime \prime}, q_{\perp}^{\prime},-q^{\prime}-q_{\mu 1} / 2\right)\right] }
\end{aligned}
$$

and in which we have defined

$\mathcal{A}\left(k_{\perp}^{\prime}, q_{\perp}, q ; q_{\mu 1}\right)=\left(2 q+\mu E(\ell)+q_{\mu 1}\right) \mathcal{B}\left(k_{\perp}^{\prime}, q_{\perp}, q ; q_{\mu 1}\right)$,

and

$$
\begin{aligned}
\mathcal{B}\left(k_{\perp}^{\prime}, q_{\perp}, q ; q_{\mu 1}\right) & \left.=\left[n_{B}\left(-\mu E_{0}(\ell)\right)-q\right)+n_{F}\left(q+q_{\mu}\right)\right] \\
& \times \frac{\left[n_{F}\left(\mu E_{0}(\ell)\right)-n_{F}(-q)\right]}{\omega_{\nu}^{2}+\left(2 q+\mu E_{0}(\ell)+q_{\mu 1}\right)^{2}},
\end{aligned}
$$

where $q_{\mu 1}=\mu E_{0}(\ell)+E_{+}(\mathbf{k})+A_{C}\left(k_{\perp}^{\prime}, q_{\perp}\right)+A_{C}\left(k_{\perp}, q_{\perp}\right)$, and $A_{C}\left(k_{\perp}, q_{\perp}\right)=\epsilon_{\perp}\left(k_{\perp}+q_{\perp}\right)-\epsilon_{\perp}\left(k_{\perp}\right)$.
The outer shell calculation of the remaining two-loop terms $\delta \Sigma_{+}^{(2,3)}$ are obtained in the same way. We only give the final results:

$$
\begin{aligned}
\delta \Sigma_{+}^{(2)}\left(\bar{k}_{\perp}\right)=\frac{1}{2} \iint \frac{d k_{\perp}^{\prime}}{2 \pi} \frac{d q_{\perp}}{2 \pi} \\
\quad \times\left(-g_{2}^{2}\left(\widetilde{k}_{\perp 2}\right)-g_{1}^{2}\left(\widetilde{k}_{\perp 2}\right)+g_{2}\left(\widetilde{k}_{\perp 2}\right) g_{1}\left(\widetilde{k}_{\perp 2}\right)\right) \\
\quad \times\left(-\left[G_{+}^{0}(\bar{k})\right]^{-1} I_{2}\left(\widetilde{k}_{\perp}, i \omega_{\nu}\right) d \ell+I_{2}^{\prime}\left(\widetilde{k}_{\perp}, i \omega_{\nu}\right) d \ell\right),
\end{aligned}
$$

$$
\begin{aligned}
\delta \Sigma_{+}^{(3)}\left(\bar{k}_{\perp}\right)=\frac{1}{2} \iint \frac{d k_{\perp}^{\prime}}{2 \pi} \frac{d q_{\perp}}{2 \pi} \\
\quad \times\left(-g_{3}^{2}\left(\widetilde{k}_{\perp 3}\right)-g_{3}^{2}\left(\widetilde{k}_{\perp 4}\right)+g_{3}\left(\widetilde{k}_{\perp 3}\right) g_{3}\left(\widetilde{k}_{\perp 4}\right)\right) \\
\quad \times\left(-\left[G_{+}^{0}(\bar{k})\right]^{-1} I_{3}\left(\widetilde{k}_{\perp}, i \omega_{\nu}\right) d \ell+I_{3}^{\prime}\left(\widetilde{k}_{\perp}, i \omega_{\nu}\right) d \ell\right)
\end{aligned}
$$

where $I_{i}^{\left({ }^{\prime}\right)}\left(\widetilde{k}_{\perp}, i \omega_{\nu}\right)=\left.I_{1}^{\left({ }^{\prime}\right)}\left(\widetilde{k}_{\perp}, i \omega_{\nu}\right)\right|_{q_{\mu 1} \rightarrow q_{\mu i}}(i=2,3)$, with

$$
\begin{aligned}
& q_{\mu 2}=\mu E_{0}(\ell)-E_{+}(\mathbf{k})+A_{C}\left(k_{\perp}^{\prime}, q_{\perp}\right)-A_{C}\left(k_{\perp},-q_{\perp}\right) \\
& q_{\mu 3}=\mu E_{0}(\ell)+E_{+}(\mathbf{k})+A_{P}\left(k_{\perp}^{\prime}, q_{\perp}\right)+A_{P}\left(k_{\perp}, q_{\perp}\right)
\end{aligned}
$$

where $A_{P}\left(k_{\perp}, q_{\perp}\right)=-\epsilon_{\perp}\left(k_{\perp}-q_{\perp}\right)-\epsilon_{\perp}\left(k_{\perp}\right)$. Finally, at the step $\ell+d \ell$, the one-particle propagator takes the form :

$$
\left[z\left(\bar{k}_{\perp}\right) G_{+}^{0}(\bar{k})\right]^{-1}+z_{\perp}\left(\bar{k}_{\perp}\right)-\left[z\left(\bar{k}_{\perp}\right)\right]^{-1} \delta \Sigma_{+}(\bar{k})
$$

There follows from Eqs. A10, A15), and (A16) the flow equations (15) and (16) of Sec. IIIB.
1 D. Jérome, A. Mazaud, M. Ribault, and K. Bechgaard, J. Phys. (Paris) Lett. 41, L95 (1980).

2 D. Jérome and H. J. Schulz, Adv. Phys. 31, 299 (1982).

3 C. Bourbonnais and D. Jérome, in The Physics of Organic Superconductors and Conductors, edited by A. Lebed (Springer, Heidelberg, 2008), vol. 110, Springer Series in Materials Science, p. 357, arXiv:cond-mat/0904.0617.

${ }^{4}$ S. E. Brown, P. M. Chaikin, and M. J. Naughton, in The Physics of Organic Superconductors and Conductors, edited by A. Lebed (Springer, Heidelberg, 2008), vol. 110, Springer Series in Materials Science, p. 49.

${ }^{5}$ C. Bourbonnais and A. Sedeki, C. R. Physique 12, 532 (2011).

6 W. Wu, P. M. Chaikin, W. Kang, J. Shinagawa, W. Yu, and S. E. Brown, Phys. Rev. Lett. 94, 097004 (2005).

7 P. Wzietek, F. Creuzet, C. Bourbonnais, D. Jérome, K. Bechgaard, and P. Batail, J. Phys. I 3, 171 (1993).

${ }^{8}$ F. Creuzet, C. Bourbonnais, L. G. Caron, D. Jérome and
A. Moradpour, Synth. Metals 19, 277 (1987); F. Creuzet, C. Bourbonnais, D. Jérome, and A. Moradpour, J. Phys. C: Solid State Phys., L821 (1985).

9 Y. Kimura, M. Misawa, and A. Kawamoto, Phys. Rev. B 84, 045123 (2011).

10 N. Doiron-Leyraud, P. Auban-Senzier, S. R. de Cotret, A. Sedeki, C. Bourbonnais, D. Jérome, K. Bechgaard, and L. Taillefer, arXiv:cond-mat/0905.0964.

11 N. Doiron-Leyraud, P. Auban-Senzier, S. R. de Cotret, C. Bourbonnais, D. Jérome, K. Bechgaard, and L. Taillefer, Phys. Rev. B 80, 214531 (2009).

12 N. Doiron-Leyraud, P. Auban-Senzier, S. R. de Cotret, C. Bourbonnais, A. Sedeki, D. Jérome, K. Bechgaard, and L. Taillefer, Eur. Phys. J. B 78, 23 (2010).

13 L. Taillefer, Annu. Rev. Condens. Matter Phys. 1, 51 (2010)

14 J. Hertz, Phys. Rev. B 14, 1165 (1976).

15 A. J. Millis, Phys. Rev. B 48, 7183 (1993). 
16 T. Moriya, Y. Takahashi, and K. Ueda, J. Phys. Soc. Jpn. 59, 2905 (1990).

17 T. Moriya and K. Ueda, Rep. Prog. Phys. 66, 1299 (2003).

18 J. C. Nickel, R. Duprat, C. Bourbonnais and N. Dupuis, Phys. Rev. Lett. 95, 247001 (2005); Phys. Rev. B 73, 165126 (2006).

19 R. Duprat and C. Bourbonnais, Eur. Phys. J. B 21, 219 (2001)

20 C. Bourbonnais and A. Sedeki, Phys. Rev. B 80, 085105 (2009)

21 K. Yamaji, J. Phys. Soc. Jpn. 51, 2787 (1982).

22 G. Montambaux, Phys. Rev. B 38, 4788 (1988).

23 J. Shinagawa, Y. Kurosaki, F. Zhang, C. Parker, S. E. Brown, D. Jérome, K. Bechgaard, and J. B. Christensen, Phys. Rev. Lett. 98, 147002 (2007).

24 C. Bourbonnais, F. Creuzet, D. Jérome, K. Bechgaard, and A. Moradpour, J. Phys. (Paris) Lett. 45, L755 (1984).

25 V. J. Emery, J. Phys. (Paris) Coll. 44, 977 (1983).

26 M. Tsuchiizu, Y. Suzumura, and C. Bourbonnais, Phys. Rev. Lett. 99, 126404 (2007).

27 D. Boies, C. Bourbonnais, and A.-M. Tremblay, Phys. Rev. Lett. 74, 968 (1995).

28 F. H. L. Essler and A. M. Tsvelik, Phys. Rev. B 65, 115117 (2002).

29 F. H. L. Essler and A. M. Tsvelik, Ann. Henri Poincaré 4, Suppl. 2, S589 (2003).

30 E. Arrigoni, Phys. Rev. Lett. 83, 128 (1999).

31 E. Arrigoni, Phys. Rev. B 61, 7909 (2000).

32 S. Biermann, A. Georges, A. Lichtenstein, and T. Giamarchi, Phys. Rev. Lett. 87, 276405 (2001).

33 C. Berthod, T. Giamarchi, S. Biermann, and A. Georges, Phys. Rev. Lett. 87, 136401 (2006).

34 P. M. Grant, Phys. Rev. B 26, 6888 (1982).

${ }^{35}$ L. Ducasse, A. Abderraba, J. Hoarau, M. Pesquer, B. Gallois, and J. Gaultier, J. Phys. C 19, 3805 (1986).

36 D. L. Pévelen, J. Gaultier, Y. Barrans, D. Chassau, F. Castet, and L. Ducasse, Eur. Phys. J. B 19, 363 (2001).

37 V. J. Emery, R. Bruinsma, and S. Barisic, Phys. Rev. Lett. 48, 1039 (1982).

${ }^{38}$ H. Nélisse, C. Bourbonnais, H. Touchette, Y. Vilk, and A.-M. S. Tremblay, Eur. Phys. J. B. 12, 351 (1999).

39 Y. Fuseya, M. Tsuchiizu, Y. Suzumura, and C. Bourbonnais, J. Phys. Soc. Jpn. 76, 014709 (2007).

40 N. Thorup, G. Rindorf, H. Soling, and K. Bechgaard, Acta Crystallogr. B 37, 1236 (1981).

41 P. M. Grant, J. Phys. (Paris) Coll. 44, 847 (1983).

42 B. J. Klemme, S. Brown, P. Wzietek, P. B. G. Kriza, D. Jérome, and J.-M. Fabre, Phys. Rev. Lett. 75, 2408 (1995).

43 C. Bourbonnais and L. G. Caron, Int. J. Mod. Phys. B 5, 1033 (1991).

44 C. Bourbonnais, B. Guay, and R. Wortis, in Theoretical methods for strongly correlated electrons, edited by D. Sénéchal, A. M. Tremblay, and C. Bourbonnais (Springer, Heidelberg, 2003), p. 77, cond-mat/0204163.

45 D. Zanchi and H. J. Schulz, Europhys. Lett. 44, 235 (1997).

46 D. Zanchi and H. J. Schulz, Phys. Rev. B 61, 13609 (2000).

47 A. Sedeki and C. Bourbonnais, unpublished results.

48 C. Honerkamp, M. Salmhofer, N. Furukawa, and T. M. Rice, Phys. Rev. B 63, 35109 (2000).

49 V. J. Emery, Synth. Met. 13, 21 (1986).

${ }^{50}$ L. G. Caron and C. Bourbonnais, Physica 143B, 453 (1986).

51 C. Bourbonnais and L. G. Caron, Europhys. Lett. 5, 209
(1988)

52 G. Chitov and C. Bourbonnais, Nucl. Phys. B 663, 568 (2003).

53 J. Solyom, Adv. Phys. 28, 201 (1979).

${ }^{54}$ T. Giamarchi, Quantum Physics in One Dimension (Oxford University Press, Oxford, 2004).

55 C. Honerkamp and M. Salmhofer, Phys. Rev. B 67, 174504 (2003).

56 C. M. Varma, P. B. Littlewood, S. Schmitt-Rink, E. Abrahams, and A. E. Ruckenstein, Phys. Rev. Lett. 63, 1996 (1989).

57 D. Bergeron, V. Hankevych, B. Kyung, and A.-M. S. Tremblay, Phys. Rev. B 84, 085128 (2011).

58 H. J. Vidberg and J. W. Serene, J. Low Temp. Phys 29, 179 (1977).

59 J. M. Luttinger, Phys. Rev. 121, 942 (1961).

60 N. Nakamura and Y. Suzumura, Prog. Theor. Phys. 97, 163 (1997).

61 N. Nakamura and Y. Suzumura, Prog. Theor. Phys. 98, 29 (1997).

62 A. Sedeki, D. Bergeron, and C. Bourbonnais, Physica B 405, S89 (2010).

63 For the same temperature interval, we have checked that the power law $\tau^{-1}=c T^{\mu}$ fits almost just as well the low temperature scattering rate than the polynomial twocomponent expression Eq. 26 In this case, the exponent $\mu$ evolves continuously from linear $(\mu=1)$ close to $t_{\perp}^{\prime *}$ toward quadratic $(\mu=2)$ at large $t_{\perp}^{\prime}$ where $T_{c} \rightarrow 0$.

64 Y. Vilk and A.-M. S. Tremblay, J. Phys. I (France) (1997).

65 A. Abanov, A. V. Chubukov, and J. Schmalian, Adv. Phys. 52, 119 (2003).

66 N. S. Vidhyadhiraja, A. Macridin, C. Sen, M. Jarrell, and M. Ma, Phys. Rev. Lett. 102, 206407 (2009).

67 P. Auban-Senzier, D. Jérome, N. Doiron-Leyraud, S. R. de Cotret, A. Sedeki, C. Bourbonnais, L. Taillefer, P. Alemany, E. Canadell, and K. Bechgaard, J. Phys. : Condens. Matter 23, 345702 (2011).

68 T. Vuletic, P. Auban-Senzier, C. Pasquier, S. Tomic, D. Jerome, M. Heritier, and K. Bechgaard, Eur. Phys. J. B 25, 319 (2002).

69 Y. Nakai, T. Iye, S. Kitagawa, K. Ishida, H. Ikeda, S. Kasahara, H. Shishido, T. Shibauchi, Y. Matsuda, and T. Terashima, Phys. Rev. Lett. 105, 107003 (2010).

${ }^{70}$ F. L. Ning, K. Ahilan, T. Imai, A. Sefat, M. A. McGuire, B. C. Sales, D. Mandrus, P. Cheng, B. Shen, and H.-H. Wen, Phys. Rev. Lett. 104, 037001 (2010).

71 F. Wang, H. Zhai, Y. Ran, A. Vishwanath, and D.-H. Lee, Phys. Rev. Lett. 102, 047005 (2009).

72 A. V. Chubukov, D. V. Efremov, and I. Eremin, Phys. Rev. B 78, 134512 (2008).

73 R. Thomale, C. Platt, J. Hu, C. Honerkamp, and B. A. Bernevig, Phys. Rev. B 80, 180505 (2009).

74 T. Manako, Y. Kubo, and Y. Shimakawa, Phys. Rev. B 46, 11019 (1992).

75 R. A. Cooper, Y. Wang, B. Vignolle, O. J. Lipscombe, S. M. Hayden, Y. Tanabe, T. Adachi, Y. Koike, M. Nohara, H. Takagi, et al., Science 323, 603 (2009).

76 R. Daou, N. Doiron-Leyraud, D. LeBoeuf, S. Y. Li, F. Laliberté, O. Cyr-Choinière, Y. J. Jo, L. Balicas, J.-Q. Tan, J.-S. Zhou, et al., Nature Physics 5, 31 (2009).

77 S. Nakamae, K. Behnia, N. Mangkorntong, M. Nohara, H. Takagi, S. C. J. Yates, and N. E. Hussey, Phys. Rev. B 68, 100502 (2003).

78 M. Ossadnik, C. Honerkamp, T. M. Rice, and M. Sigrist, 
Phys. Rev. Lett. 101, 256405 (2008).

79 N. P. Armitage, P. Fournier, and R. L. Greene, Rev. Mod. Phys. 82, 2421 (2010).

80 B. Kyung, J.-S. Landry, and A. M. S. Tremblay, Phys. Rev. B 68, 174502 (2003).

${ }^{81}$ S. R. Hassan, B. Davoudi, B. Kyung, and A.-M. S. Tremblay, Phys. Rev. B 77, 094501 (2008).

${ }^{82}$ P. Fournier, P. Mohanty, E. Maiser, S. Darzens, T. Venkatesan, C. J. Lobb, G. Czjzek, R. A. Webb, and R. L. Greene, Phys. Rev. Lett. 81, 4720 (1998).

${ }^{83}$ K. Jin, N. P. Butch, K. Kirshenbaum, J. Paglione, and R. L. Greene, Nature 476, 73 (2011).

${ }^{84}$ C. Honerkamp, Eur. Phys. J. B 21, 81 (2001).

85 S. A. Hartnoll, D. M. Hofman, M. A. Metlitski, and S. Sachdev, Phys. Rev. B 84, 125115 (2011).

${ }^{86}$ M. A. Metlitski and S. Sachdev, Phys. Rev. B 82, 075128
(2010).

87 M. Abdel-Jawad, M. P. Kenett, A. C. L. Balicas, A. P. Mackenzie, R. H. Mackenzie, and N. E. Hussey, Nature Phys. 2, 821 (2006).

${ }^{88}$ M. Abdel-Jawad, J. G. Analytis, L. Balicas, A. Carrington, J. P. H. Charmant, M. M. J. French, and N. E. Hussey, Phys. Rev. Lett. 99, 107002 (2007).

89 A. A. Katanin and A. P. Kampf, Phys. Rev. Lett. 93, 106406 (2004).

90 D. Rohe and W.Metzner, Phys. Rev. B 71, 115116 (2005).

91 H. Freire, E. Corrêa, and A. Ferraz, Phys. Rev. B 71, 165113 (2005).

${ }^{92}$ M. Tsuchiizu, Phys. Rev. B 74, 155109 (2006).

93 H. Freire, E. Correa, and A. Ferraz, Phys. Rev. B 78, 125114 (2008). 